\title{
On Essential Selfadjointness, Distinguished Selfadjoint Extension and Essential Spectrum of Dirac Operators with Matrix Valued Potentials
}

By

\author{
Masaharu ARAI*
}

\section{$\S 1$. Introduction}

The Dirac operator with a $4 \times 4$ symmetric (i.e. Hermitian symmetric) matrix valued measurable potential $Q(x)$ is given by

$$
H=\sum_{j=1}^{3} \alpha_{j} p_{j}+\beta+Q(x) \quad\left(x \in \boldsymbol{R}^{3}\right),
$$

where $p_{j}=-i \partial / \partial x_{j}(i=\sqrt{-1})$, and $\alpha_{j}, \beta=\alpha_{4}$ are $4 \times 4$ constant symmetric matrices satisfying the anti-commutation relations

$$
\alpha_{j} \alpha_{k}+\alpha_{k} \alpha_{j}=2 \delta_{j k} I \quad(j, k=1,2,3,4) .
$$

We denote by $H_{0}$ the operator $H$ with $Q(x) \equiv 0$.

We denote by $\langle$,$\rangle and ||$ the usual inner product and norm in $\mathbb{C}^{4}$, respectively, and by (, ) and \|\| the inner product and norm in the Hilbert space $\mathscr{H}=\left[L_{2}\left(\boldsymbol{R}^{3}\right)\right]^{4}$, respectively. We also denote by || and || || the operator norm of a $4 \times 4$ matrix and a bounded linear operator in $\mathscr{H}$, respectively. We denote by $I$ the $4 \times 4$ identity matrix, which at times implies the $2 \times 2$ identity matrix, but no confusion will occur. For a closable operator $T$ in $\mathscr{H}$, we denote by $\bar{T}$ its closure. For an (formal) operator $T$, we denote by $\dot{T}$ the restriction of $T$ to the domain

$$
\dot{D}=\left[C_{0}^{\infty}\left(\mathbb{R}^{3} \backslash O\right)\right]^{4},
$$

except $\dot{L}(k)$ defined in Section 2. It is evident that the operator $\dot{H}$ is symmetric in $\mathscr{H}$ if

$$
|Q(x)| \in L_{2,10 \mathrm{c}}\left(\mathbb{R}^{3} \backslash O\right) .
$$

We shall consider the following problems with special emphasis on (P.1) and (P. 4).

(P. 1) Is $\dot{H}$ essentially selfadjoint?

(P.2) If (P.1) is affirmatively answered, dose the domain of the unique selfadjoint extension of $\dot{H}$ coincide with that of $\dot{H}_{0}$ ? If this is true we have

Communicated by S. Matsuura, June 12, 1981.

* Faculty of Economics, Ritsumeikan University, Kyoto 603, Japan. 


$$
\mathscr{D}(\overline{\dot{H}})=\mathscr{D}\left(\bar{H}_{0}\right)=\text { the Sobolev space }\left[H^{1}\right]^{4} \text {. }
$$

(P.3) If (P. 1) is negatively answered, does $\dot{H}$ has any selfadjoint extension ?

(P.4) If (P. 3) is affirmatively answered, can we select a selfadjoint extension which has a certain clear physical meaning among selfadjoint extensions of $\dot{H}$ ? (This extension will be called distinguished after Schmincke [14], Wüst [18] [19] and Nenciu [9].)

(P.5) Does the essential spectrum of the unique selfadjoint extension or the distinguished selfadjoint extension, if any, coincide with that of $\dot{H}_{0}$ ?

Remark 1.1. One may consider, instead of $\dot{H}$, the restriction $H$ to $\mathscr{D}=$ $\left[C_{0}^{\infty}\left(\boldsymbol{R}^{3}\right)\right]^{4}$, which will be denoted by $\stackrel{\circ}{H}$ for the time being. Even if we replace $\dot{H}$ by $\stackrel{H}{H}$ in the above problems, nothing new will occur if

$$
|Q(x)| \in L_{2,10 c}\left(\boldsymbol{R}^{3}\right),
$$

which will be assumed throughout this paper. Indeed, under the assumption $(1.4)^{\prime}$, the operators $\dot{H}$ and $\dot{H}^{\circ}$ are symmetric in $\mathscr{H}$ and their closures coincide with each other. In order to prove this, it is sufficient to prove $\stackrel{H}{ } \subset \dot{H}$. The rest is obvious. Let $\phi$ be a real valued $C^{\infty}$ function in $\boldsymbol{R}^{3}$, which vanishes in $|x| \leqq 1$ and equals 1 in $|x| \geqq 2$. Put $\phi_{\varepsilon}(x)=\phi(x / \varepsilon)$. Let $\phi \in \mathscr{D}$. $\phi_{\varepsilon} \psi \in \dot{D}$ tends strongly to $\psi$ in $\mathscr{H}$ as $\varepsilon \downarrow 0$.

$$
\dot{H}\left(\phi_{\varepsilon} \psi\right)=-i \frac{1}{\varepsilon} \sum_{j=1}^{3} \alpha_{j} \frac{\partial \phi}{\partial x_{j}}(x / \varepsilon) \cdot \psi+\phi_{\varepsilon} \dot{H} \psi .
$$

The $\mathscr{H}$-norm of the first term in the right hand side of the above equation is of order $\varepsilon^{1 / 2}$, since $\psi$ is bounded, so that it vanishes as $\varepsilon \downarrow 0$. The second term tends to $\stackrel{H}{\phi} \phi$, which yields $\stackrel{H}{ } \subset \bar{H}$.

Let us sketch some results already known and what our main interests are in connection with (P.1). At first we note that only the local behavior of $Q(x)$ affects (P.1):

Lemma 1.2 (Chernoff [3]). Assume that for any $y \in \boldsymbol{R}^{3}$, there exists a potential $Q_{y}(\cdot) \in L_{2, \text { loc }}$ such that $Q(x)=Q_{y}(x)$ in some neighborhood of $y$ and that $\dot{H}_{0}+Q_{y}$ is essentially selfadjoint. Then $\dot{H}=\dot{H}_{0}+Q$ is also essentially selfadjoint.

Thus our consideration will be concentrated on the local singularities of $Q(x)$. But if the singularities are so weak that for any constant $a>0$ there exists a constant $b$ such that

$$
\|Q u\| \leqq a\left\|H_{0} u\right\|+b\|u\| \quad \text { for any } u \in \dot{\mathscr{D}},
$$

then we can apply the following well-known theorem with $T_{0}=\dot{H}_{0}$ and $V=Q$ to see that (P.1) and (P.2) are affirmatively answered. (As to sufficient conditions for (1.6) see $[2 ; \S 2$.$] )$ 
Lemma 1.3 (Kato [5]; Theorems 4.4 and 4.6 in Chap. V). Let $T_{0}$ be an essentially selfadjoint operator and $V$ be a symmetric operator such that $\mathscr{D}\left(T_{0}\right) \subset$ $\mathscr{D}(V)$. Put $T=T_{0}+V$.

(1) Assume that there exist positive constants $a$ and $b$ such that $a \leqq 1$ and (1.7)

$$
\|V u\| \leqq a\left\|T_{0} u\right\|+b\|u\| \quad \text { for any } u \in \mathscr{D}\left(T_{0}\right) .
$$

Then $T$ is essentially selfadjoint.

(2) If in addition $a<1$, then $\mathscr{D}(\bar{T})=\mathscr{D}\left(\overline{T_{0}}\right)$.

Let $Q(x)=r^{-\alpha} I \quad\left(r=\left(\sum_{j=1}^{3} x_{j}^{2}\right)^{1 / 2}, \alpha\right.$ : positive constant $)$. Then each sufficient condition for (1.6) listed up in [2] requires the same condition $\alpha<1$. Thus the simplest case to be considered next is

$$
Q(x)=\frac{e}{r} I \quad(e: \text { constant }) .
$$

Kato ([5]; p. 307) shows that $\dot{H}$ is essentially selfadjoint if $|e| \leqq 1 / 2$, using the well-known inequality

$$
\int \frac{1}{r^{2}}|u|^{2} d x \leqq 4 \int \sum_{j=1}^{3}\left|\frac{\partial u}{\partial x_{j}}\right|^{2} d x \leqq 4\left\|H_{0} u\right\|^{2} \quad(\forall u \in \dot{D})
$$

and Lemma 1.3. Since this result is based on (1.7) and (1.9), we can easily extend to the case when the potential $Q(x)$ is matrix valued, that is, we have if

$$
\sup _{x} r|Q(x)|=e \leqq 1 / 2 \text {, }
$$

then $\dot{H}$ is essentially selfadjoint. Moreover, if $e<1 / 2$, then (1.5) holds.

Now we return to the case (1.8). Weidmann [17] (see also Rellich [10] [11]) shows by separating out the angular variables that $\dot{H}$ is essentially selfadjoint if and only if $|e| \leqq \sqrt{3} / 2$. The "if part" of this assertion is extended to the case of a scalar potential

$$
Q(x)=q(x) I
$$

by Schmincke [13], who shows that the assumption

$$
\sup _{x} r|q(x)|<\sqrt{3} / 2
$$

implies the essential selfadjointness of $\dot{H}$ and (1.5). (Strictly speaking, his assumption is more general than (1.12) and he does not state explicitly (1.5).)

Since Kato's result is extended to the case of matrix potentials, and RellichWeidmann's to the case of a scalar potential (1.11) as above, one might expect that, also in the case of matrix potentials, the assumption $\sup _{x} r|Q(x)|<\sqrt{3} / 2$ is sufficient for $\dot{H}$ to be essentially selfadjoint. But this is not true. On the contrary, Arai [1] showed that the number $1 / 2$ in the assumption (1.10) is best possible in the following sense.

Theorem 1.4. For any constant $e>1 / 2$, there exists a matrix valued potential 
$Q(x)$ with

$$
|Q(x)|=e / r
$$

such that $\dot{H}$ is not essentially selfadjoint and $\dot{H}$ has selfadjoint extensions.

This will be re-proved in Section 2 .

Remark 1.5. It is also true that for any $e \geqq 0$ there exists a potential $Q(x)$ satisfying (1.13) such that $\dot{H}$ is essentially selfadjoint. This is easy to see by putting $Q \equiv 0$ and $g=e \log r$ in the next lemma.

Lemma 1.6. Let $g(x)$ be a real valued $C^{\infty}$ function in $\boldsymbol{R}^{3} \backslash O$. The operator $\dot{H}$ with the potential $Q(x)$ is unitarily equivalent to the operator $\dot{H}_{1}$ with the potential $Q_{1}(x) \equiv Q(x)-\sum_{j} \alpha_{j} \frac{\partial g}{\partial x_{j}}$ by means of the unitary operator of multiplication by $\exp [-i g(x)]$.

Recently, Arai and Yamada [2] have shown the following result: Put

$$
V(x)=Q(x)-i \alpha_{r} /(2 r) \quad\left(\alpha_{r}=\sum_{j=1}^{3} \alpha_{j} x_{j} / r\right),
$$

and assume that there exists a constant $m_{0}$ such that

$$
r^{2} V(x) * V(x) \leqq m_{0}<1 \text {. }
$$

Then $\dot{H}$ is essentially selfadjoint and (1.15) holds.

Note that in the case of (1.11), (1.14) is reduced to (1.12).

In Section 2, we shall treat a certain class of potentials which allows us to apply the separation of variables and we shall give a necessary and sufficient condition for these $\dot{H}$ 's to be essentially selfadjoint (Theorem 2.7 ) and the proof of Theorem 1.4. We shall also consider (P. 3) there (Theorem 2.9). In Section 3, we shall discuss (P.1) and (P.2) for more general potentials and generalize the above mentioned result of Arai-Yamada [2] (Theorem 3.1). In Section 4, we shall treat (P.4) and (P.5) (Theorem 4.1).

\section{$\S 2 . \quad$ Separation of Variables}

Almost all lemmas stated in this section are well known. Some of them will be proved here for the sake of completeness.

We define the matrices $\sigma_{l}^{\prime}(l=1,2,3)$ by

$$
\sigma_{l}^{\prime}=-i \alpha_{\jmath} \alpha_{k} \quad(j, k, l)=(1,2,3) \text { in the cyclic order. }
$$

Then they are symmetric and unitary and satisfy

$$
\begin{cases}\text { (a) } \quad \sigma_{\jmath}^{\prime} \sigma_{k}^{\prime}=i \sigma_{l}^{\prime} & (j, k, l)=(1,2,3) \text { in the cyclic order } \\ \text { (b) } \quad \sigma_{\jmath}^{\prime} \sigma_{k}^{\prime}+\sigma_{k}^{\prime} \sigma_{\jmath}^{\prime}=2 \delta_{\jmath k} & (j, k=1,2,3), \\ \text { (c) } \quad \sigma_{\jmath}^{\prime} \beta=\beta \sigma_{j}^{\prime} & (j=1,2,3) .\end{cases}
$$


Lemma 2.1. There exists an orthonormal basis of $C^{4}$ in terms of which $\alpha_{j}$, $\sigma_{j}^{\prime}$ and $\beta$ are represented as follows:

$$
\alpha_{j}=\left(\begin{array}{cc}
0 & \sigma_{\jmath} \\
\sigma_{j} & 0
\end{array}\right), \quad \sigma_{j}^{\prime}=\left(\begin{array}{cc}
\sigma_{\jmath} & 0 \\
0 & \sigma_{j}
\end{array}\right), \quad \beta=\left(\begin{array}{cc}
I & 0 \\
0 & -I
\end{array}\right), \quad(j=1,2,3),
$$

where

$$
\sigma_{1}=\left(\begin{array}{ll}
0 & 1 \\
1 & 0
\end{array}\right), \quad \sigma_{2}=\left(\begin{array}{rr}
0 & -i \\
i & 0
\end{array}\right), \quad \sigma_{3}=\left(\begin{array}{rr}
1 & 0 \\
0 & -1
\end{array}\right)
$$

Proof. (1.2) with $j=k=4$ implies $\beta^{2}=I$ so that the eigenvalues of $\beta$ are \pm 1 , whose eigenspaces will be denoted by $\mathbb{C}( \pm)$. Again, (1.2) implies that $\alpha_{1}$ maps $\boldsymbol{C}( \pm)$ onto $\boldsymbol{C}(\mp)$ bijectively so that $\operatorname{dim} \boldsymbol{C}(+)=\operatorname{dim} \boldsymbol{C}(-)=2$. The eigenspaces $\boldsymbol{C}( \pm)$ reduce $\sigma_{j}^{\prime}$ by virtue of $(2.2-\mathrm{c})$. We denote by $\sigma_{j}$ the part of $\sigma_{j}^{\prime}$ in $\mathbb{C}(+)$. Then (2.2) implies

$$
\begin{cases}\text { (a) } \quad \sigma_{j} \sigma_{k}=i \sigma_{l} & (j, k, l)=(1,2,3) \text { in cyclic order, } \\ \text { (b) } \quad \sigma_{j} \sigma_{k}+\sigma_{k} \sigma_{j}=2 \delta_{j k} & (j, k=1,2,3) .\end{cases}
$$

The same argument as above shows that the eigenvalues of $\sigma_{3}$ are \pm 1 and its eigenspaces are of dimension 1 and are mapped by $\sigma_{1}$ onto each other. Let $\vec{e}_{1}$ be a normalized eigenvector of $\sigma_{3}$ belonging to +1 and $\vec{e}_{2}=\sigma_{1} \vec{e}_{1}$, which is a normalized eigenvector of $\sigma_{3}$ belonging to -1 . In this co-ordinate system, the $\sigma_{j}$ 's are represented as (2.4), where we have used (2.2-a) to obtain the representation of $\sigma_{2}$ from the others. We define $\rho \equiv-i \alpha_{1} \alpha_{2} \alpha_{3}=\rho^{-1}$, which is symmetric and unitary, and maps $C( \pm)$ onto $C(\mp)$. (1.2) and (2.1) imply

$$
\alpha_{j}=\rho \sigma_{j}^{\prime}=\sigma_{j}^{\prime} \rho \text {. }
$$

Put $\vec{e}_{3}=\rho \vec{e}_{1}$ and $\vec{e}_{4}=\rho \vec{e}_{2}$. Then $\left\{\vec{e}_{1}, \vec{e}_{2}, \vec{e}_{3}, \vec{e}_{4}\right\}$ is an orthonormal basis of $\mathbb{C}^{4}$. It is obvious that $\beta$ is represented as in (2.3) and that

$$
\rho=\left(\begin{array}{ll}
0 & I \\
I & 0
\end{array}\right)
$$

in terms of this basis. (2.5) and (2.6) yield the representations of $\alpha_{j}$ and $\sigma_{j}^{\prime}$ in (2.3), since $\boldsymbol{C}( \pm)$ reduce $\sigma_{j}^{\prime}$.

We put $\vec{\alpha}=\left(\alpha_{1}, \alpha_{2}, \alpha_{3}\right), \vec{x}=\left(x_{1}, x_{2}, x_{3}\right), \vec{p}=\left(p_{1}, p_{2}, p_{3}\right)$,

$$
\begin{aligned}
\alpha_{r}=\vec{\alpha} \cdot \vec{x} / r, \quad p_{r} & =r^{-1}(\vec{x} \cdot \vec{p}-i)=-i(\partial / \partial r+1 / r), \\
\vec{L}=\vec{x} \times \vec{p} & =\left(L_{1}, L_{2}, L_{3}\right)
\end{aligned}
$$

and

$$
K=\beta\left(\vec{\sigma}^{\prime} \cdot \vec{L}+I\right) .
$$

Then $L_{j}$ commutes with the multiplication operator $r \times$, and $K$ with $H_{0}, \alpha, \beta$, $p_{r}$ and $r \times$ at least in $\dot{D}$. We regard the Hilbert space $\mathscr{H}$ as

$$
\mathscr{H}=\boldsymbol{C}^{4} \otimes L_{2}\left(\mathbb{R}^{3}\right)=\boldsymbol{C}^{4} \otimes L_{2}\left(S^{2}\right) \otimes L_{2}\left(\boldsymbol{R}_{+} ; r^{2} d r\right),
$$


where $S^{2}$ denote the unit sphere in $\boldsymbol{R}^{3}$ and $\boldsymbol{R}_{+}=(0, \infty)$. Then $\vec{\alpha}, \alpha, \beta, \vec{\sigma}^{\prime}, \vec{L}$ and $K$ can be regarded as operators in the Hilbert space $\mathfrak{S} \equiv C^{4} \otimes L_{2}\left(S^{2}\right)$.

Lemma 2.2. The Hilbert space $\mathfrak{S}$ can be decomposed into an orthogonal direct sum of two-dimensional spaces $\mathfrak{S}(k, m)(k= \pm 1, \pm 2, \cdots$ and $m=-|k|,-|k|+1$, $\cdots,|k|-1)$ :

$$
\mathfrak{H}=\sum_{k, m} \oplus \mathfrak{S}(k, m)
$$

such that

(i) each $\mathfrak{S}(k, m)$ reduces $\alpha_{r}, \beta$ and $K$,

(ii) $K=k \times$ on $\mathfrak{S}(k, m)$,

(iii) each $\mathfrak{S}(k, m)$ has an orthonormal basis $\left\{\Phi^{( \pm)}(k, m)\right\}$ consisting of $C^{4}$ valued $C^{\infty}$ functions on $S^{2}$, in terms of which $\alpha_{r}$ and $\beta$ are represented as

$$
\alpha_{r}=\left(\begin{array}{rr}
0 & -i \\
i & 0
\end{array}\right), \quad \beta=\left(\begin{array}{rr}
1 & 0 \\
0 & -1
\end{array}\right) \text {. }
$$

Proof. Let $\{Y(l, m) ; l=0,1,2, \cdots$ and $m=-l,-l+1, \cdots, l\}$ be the totality of the spherical harmonic functions, which is a complete orthonormal system of $L_{2}\left(S^{2}\right)$, so that we have the decomposition

$$
\mathfrak{S}=\sum_{l=0}^{\infty} \oplus\{[C(+) \otimes \mathscr{g}(l)] \oplus[C(-) \otimes \mathscr{g}(l)]\},
$$

where $C( \pm)$ are those defined in the proof of Lemma 2.1, and $y(l)$ is the linear hull of $\{Y(l, m) ; m=-l,-l+1, \cdots, l\}$ for each fixed $l$. We adopt the same basis as in the proof of Lemma 2.1 for $C( \pm)$ and $C^{4}$. The image of the general element $\sum_{m}\left(\begin{array}{l}C_{1, m} \\ C_{2, m}\end{array}\right) Y(l, m)$ in $C(+) \otimes y(l)$ by the operator $\vec{\sigma} \cdot \vec{L}+I$ is

$$
\sum_{m}\left(\begin{array}{l}
\sqrt{(l+m+1)(l-m)} C_{2, m+1}+(m+1) C_{1, m} \\
\sqrt{(l-m+1)(l+m)} C_{1, m-1}+(1-m) C_{2, m}
\end{array}\right) Y(l, m),
$$

where we used (2.4), the well-known identities

$$
\left\{\begin{array}{l}
L_{1} Y(l, m) \\
=\frac{1}{2}[\sqrt{(l+m+1)(l-m)} Y(l, m+1)+\sqrt{(l-m+1)(l+m)} Y(l, m-1)] \\
L_{2} Y(l, m) \\
=\frac{1}{2 i}[\sqrt{(l+m+1)(l-m)} Y(l, m+1)-\sqrt{(l-m+1)(l+m)} Y(l, m-1)] \\
L_{3} Y(l, m)=m Y(l, m)
\end{array}\right.
$$

and conventions $Y(l, m)=0, \quad C_{i, m}=0$ for $|m|>l$. Thus $\vec{\sigma} \cdot \vec{L}+I$ restricted to $C(+) \otimes y(l)$ has an eigenvalue $l+1$ with orthonormal $2 l+2$ eigenvectors

$$
\frac{1}{\sqrt{2 l+1}}\left(\begin{array}{l}
\sqrt{l+m+1} Y(l, m) \\
\sqrt{l-m} Y(l, m+1)
\end{array}\right) \quad(m=-l-1,-l, \cdots, l-1, l)
$$


and the other eigenvalue $-l$ with $2 l$ orthonormal eigenvectors

$$
\frac{1}{\sqrt{2 l+1}}\left(\begin{array}{l}
-\sqrt{l-m} Y(l, m) \\
\sqrt{l+m+1} Y(l, m+1)
\end{array}\right) \quad(m=-l,-l+1, \cdots, l-1) .
$$

For $k= \pm 1, \pm 2, \cdots$ and $m=-|k|, \cdots,|k|-1$, we put

$$
\phi(k, m)= \begin{cases}\frac{1}{\sqrt{2 k-1}}\left(\begin{array}{l}
\sqrt{k+m} Y(k-1, m) \\
\sqrt{k-m-1} Y(k-1, m+1)
\end{array}\right) & (k>0) \\
\frac{C_{k, m}}{\sqrt{2|k|+1}}\left(\begin{array}{l}
-\sqrt{|k|-m} Y(|k|, m) \\
\sqrt{|k|+m+1} Y(|k|, m+1)
\end{array}\right) & (k>0),\end{cases}
$$

where $C_{k, m}$ are constants with modulus one, which will be determined later. Then we have

$$
(\vec{\sigma} \cdot \vec{L}+I) \psi(k, m)=k \phi(k, m) .
$$

An elementary calculation shows the anti-commutation relation

$$
\sigma_{r}(\vec{\sigma} \cdot \vec{L}+I)=-(\vec{\sigma} \cdot \vec{L}+I) \sigma_{r} \quad\left(\sigma_{r}=\vec{\sigma} \cdot \vec{x} / r\right),
$$

which implies that $\sigma_{r} \psi(k, m)$ is a linear combination of $\phi\left(-k, m^{\prime}\right)$ with fixed $k$. On the other hand it is shown that the first component of $\sigma_{r} \psi(k, m)$ is an eigenfunction of $L_{3}$ belonging to the eigenvalue $m$, so that we have $\sigma_{r} \psi(k, m)$ $=\widetilde{C}_{k, m} \psi(-k, m)$, where $\widetilde{C}_{k, m}$ is a constant with modulus 1 . We choose constants $C_{k, m}$ in (2.15) so as to yield $\tilde{C}_{k, m}=-i$ for $k>0$. Then we have

$$
\sigma_{r} \psi(k, m)=-i(\operatorname{sgn} k) \psi(-k, m)
$$

by virtue of $\sigma_{r}{ }^{2}=I$. We put

$$
\Phi^{(+)}(k, m)=\left(\begin{array}{c}
\psi(k, m) \\
0 \\
0
\end{array}\right), \quad \Phi^{(-)}(k, m)=\left(\begin{array}{c}
0 \\
0 \\
(\operatorname{sgn} k) \phi(-k, m)
\end{array}\right)
$$

and let $\mathfrak{S}(k, m)$ be the linear hull of $\Phi^{( \pm)}(k, m)$ with fixed $k$ and $m$. The above arguments show that the $\mathfrak{S}(k, m)$ 's satisfy the required properties.

Now, since

$$
\begin{aligned}
\vec{\alpha} \cdot \vec{p} & =\alpha_{r}\left\{\sum_{j, k=1}^{3} r^{-1} x_{\jmath} \alpha_{\jmath} \alpha_{k} p_{k}\right\} \\
& =\alpha_{r}\left\{r^{-1} \vec{x} \cdot \vec{p}+i r^{-1} \sum_{l=1}^{3} \sigma_{l}^{\prime}(\vec{x} \times \vec{p})_{l}\right\} \\
& =\alpha_{r}\left\{p_{r}+i r^{-1} \beta K\right\}
\end{aligned}
$$

we have

$$
H_{0}=\alpha_{r} p_{r}+i r^{-1} \alpha_{r} \beta K+\beta,
$$

which is reduced by $\mathscr{T}(k, m) \equiv \mathfrak{S}(k, m) \otimes L_{2}\left(\boldsymbol{R}_{+} ; r^{2} d r\right)$ by virtue of Lemma 2.2 (i). Let $Q(x)$ be a linear combination of $I, \alpha_{r}, \beta$ and $i \alpha_{r} \beta$ with coefficients which 
are functions of $r$ only. Then $H$ is also reduced by $\mathscr{H}(k, m)$. But the term $f(r) \alpha_{r}$ has no influence on (P.1) by Lemma 1.6. (Let $g=g(r)$ be a primitive function of $f(r)$.) Our central interest is in the potential $Q(x)$ satisfying (1.13). Hence we assume

$$
Q(x)=\frac{a}{r} I+\frac{i}{r} \alpha_{r} \beta b_{1}+\frac{\beta}{r} b_{2} \quad\left(a, b_{1}, b_{2}: \text { real constants }\right)
$$

in this section. Since we consider (P.1), we may omit the bounded operator $\beta$ from $H$ and assume

$$
H=\alpha_{r} p_{r}+\frac{i}{r} \alpha_{r} \beta K+\frac{a}{r}+\frac{i}{r} \alpha_{r} \beta b_{1}+\frac{\beta}{r} b_{2} .
$$

An element $u$ in $\mathscr{H}(k, m)$ can be expressed as

$$
\begin{aligned}
u & =\frac{1}{r} \phi_{1}(r) \Phi^{(+)}(k, m)+\frac{1}{r} \phi_{2}(r) \Phi^{(-)}(k, m) \\
& \equiv U_{k, m}(\phi), \quad \phi={ }^{t}\left(\phi_{1}, \phi_{2}\right) \in\left[L_{2}\left(\boldsymbol{R}_{+}\right)\right]^{2},
\end{aligned}
$$

and $H$ in this reducing subspace becomes

$$
L(k)\left(\begin{array}{c}
\phi_{1} \\
\phi_{2}
\end{array}\right)=\left(\begin{array}{c}
-\phi_{2}{ }^{\prime}+\frac{a+b_{2}}{r} \phi_{1}-\frac{k+b_{1}}{r} \phi_{2} \\
\phi_{1}{ }^{\prime}+\frac{a-b_{2}}{r} \phi_{2}-\frac{k+b_{1}}{r} \phi_{1}
\end{array}\right) \quad\left({ }^{\prime}=d / d r\right),
$$

where we used (2.7), Lemma 2.2(ii) and (2.12). We denote by $\dot{L}(k)$ the restriction of $L(k)$ to $\dot{\mathscr{D}}_{1} \equiv\left[C_{0}^{\infty}\left(\boldsymbol{R}_{+}\right)\right]^{2}$. Each $\dot{L}(k)$ is a symmetric operator in the Hilbert space $\left[L_{2}\left(\boldsymbol{R}_{+}\right)\right]^{2}$. Since $\Phi^{( \pm)}(k, m) \in C^{\infty}\left(S^{2}\right)$ by Lemma 2.2 (iii), the orthogonal projection $P_{k, m}$ onto $\mathscr{H}(k, m)$ maps $\dot{D}$ onto the totality of the functions $u=$ $U_{k, m}(\phi), \phi \in \dot{D}_{1}$.

Lemma 2.3. $\dot{H}$ is essentially selfadjoint if and only if all $\dot{L}(k)$ are essentially selfadjoint.

Proof. Assume that some $\dot{L}(k)$ is not essentially selfadjoint. Then there exists a non-trivial vector $\psi \in\left[L_{2}\left(\boldsymbol{R}_{+}\right)\right]^{2}$ satisfying

$$
(\dot{L}(k) \phi, \phi)=i(\phi, \phi) \quad \text { for any } \phi \in \dot{\mathscr{D}}_{1} \text {. }
$$

We put $v=U_{k, m}(\psi)$. Let $\tilde{\phi} \in \dot{D}$ and put $u=P_{k, m} \tilde{\phi}=U_{k, m}(\phi)$. Then $\phi \in \dot{D}_{1}$. We have $(\dot{H} \tilde{\phi}, v)=\left(P_{k, m} \dot{H} \tilde{\phi}, v\right)=\left(\dot{H} P_{k, m} \tilde{\phi}, v\right)=(\dot{L}(k) \phi, \phi)=i(\phi, \phi)=i(u, v)=i\left(P_{k, m} \tilde{\phi}, v\right)$ $=i(\tilde{\phi}, v)$ for any $\tilde{\phi} \in \dot{\mathcal{D}}$, which implies that $\dot{H}$ is not essentially selfadjoint.

Conversely, assume that $\dot{H}$ is not essentially selfadjoint. Then there exists a non-trivial vector $\tilde{\psi} \in \mathscr{H}$ such that

$$
(\dot{H} \tilde{\phi}, \tilde{\phi})=i(\tilde{\phi}, \tilde{\phi}) \quad \text { for any } \quad \tilde{\phi} \in \dot{\mathscr{D}} \text {. }
$$

There exists $(k, m)$ such that $v \equiv P_{k, m} \tilde{\psi}=U_{k, m}(\phi) \neq 0$. Let $\tilde{\phi}=U_{k, m}(\phi), \phi \in \dot{D}_{1}$. Then (2.25) reduces to (2.24), which shows that this $\dot{L}(k)$ is not essentially selfadjoint. 
As to the operator $L(k)$, the analogue of Weyl's alternative theorem on the second order differential euqations holds :

Lemma 2.4 ([17]; Sätze 1.4 and 1.5, see also [12]). i) Assume that for some $\lambda \in C$ all solutions of the equation

$$
L(k) \phi=\lambda \phi \quad(k: \text { fixed })
$$

satisfy

$$
\int_{0}^{1}|\phi|^{2} d r<\infty .
$$

Then for any $\lambda \in C$ all solutions of (2.26) also satisfy (2.27). (In this case we say that $L(k)$ is in the limit circle case at 0 , and otherwise, in the limit point case at 0.$)$

i)' The above assertion is also true when the condition $(2.27)_{0}$ is replaced by

$$
\int_{1}^{\infty}|\phi|^{2} d r<\infty
$$

(We define similarly "limit circle case at $\infty$ " and "limit point case at $\infty$ ".)

ii) For any non-real $\lambda,(2.26)$ has at least one non-trivial solution satisfying $(2.27)_{0}$ and also has at least one non-trivial solution satisfying $(2.27)_{\infty}$.

iii) The operator $\dot{L}(k)$ is essentially selfadjoint if and only if $L(k)$ is in the limit point case at both end points 0 and $\infty$.

Lemma 2.5 (Evans [4]; p. 538, Weidmann [17]; Satz 5.1). $L(k)$ is in the limit point case at $\infty$.

Combining the above three lemmas, we have

Corollary 2.6. $\dot{H}$ defined by (2.21) is essentially selfadjoint if and only if all the equations

$$
L(k) \phi=0 \quad(k= \pm 1, \pm 2, \cdots)
$$

have at least one solution which does not satisfy $(2.27)_{0}$.

Now, let us solve (2.28). We put $\rho=\left(k+b_{1}\right)^{2}-a^{2}+b_{2}{ }^{2}$ and $s_{ \pm}=k+b_{1} \pm \sqrt{\rho}$. Direct calculation shows that the following pairs are systems of linearly independent solutions of (2.28).

(i) $\phi=r^{\mp \sqrt{\rho}}\left(\begin{array}{c}a-b_{2} \\ s_{ \pm}\end{array}\right)$if $\rho \neq 0$ and $a \neq b_{2}$,

(ii) $\phi=r^{\mp \sqrt{\rho}}\left(\begin{array}{c}s_{\mp} \\ a+b_{2}\end{array}\right)$ if $\rho \neq 0$ and $a+b_{2} \neq 0$,

(iii) $\dot{\phi}=\left(\begin{array}{l}a-b_{2} \\ k+b_{1}\end{array}\right),\left(\begin{array}{ll}\left(a-b_{2}\right) \log r & \\ \left(k+b_{1}\right) \log r & -1\end{array}\right) \quad$ if $\rho=0$ and $a \neq b_{2}$, 
(iv) $\phi=\left(\begin{array}{l}k+b_{1} \\ a+b_{2}\end{array}\right),\left(\begin{array}{l}\left(k+b_{1}\right) \log r \\ \left(a+b_{2}\right) \log r\end{array}\right) \quad$ if $\rho=0$ and $a+b_{2} \div 0$,

(v) $\phi=\left(\begin{array}{c}r^{k+b_{1}} \\ 0\end{array}\right),\left(\begin{array}{c}0 \\ r^{-\left(k+b_{1}\right)}\end{array}\right) \quad$ if $a=b_{2}=0$.

Thus, $L(k)$ is in the limit point case at 0 if and only if $|\operatorname{Re} \sqrt{\rho}| \geqq 1 / 2$, which is equivalent to

$$
\left(k+b_{1}\right)^{2}+{b_{2}}^{2} \geqq a^{2}+1 / 4
$$

Summing up, we have

Theorem 2.7. The operator $\dot{H}$ with $Q$ given by (2.20) is essentially selfadjoint if and only if (2.29) holds for all $k= \pm 1, \pm 2, \cdots$.

Let $b_{1}=b_{2}=0$ in this theorem. Then we have the result of Weidmann [17] mentioned in Section 1.

Proof of Theorem 1.4. Since $\left(i \alpha_{r} \beta b_{1}+\beta b_{2}\right)^{2}=\left(b_{1}{ }^{2}+b_{2}{ }^{2}\right) I$ and $i \alpha_{r} \beta b_{1}+\beta b_{2}$ is not a scalar times $I$ if ${b_{1}}^{2}+{b_{2}}^{2} \neq 0$, the eigenvalues of $i \alpha_{r} \beta b_{1}+\beta b_{2}$ are $\pm \sqrt{b_{1}{ }^{2}+b_{2}{ }^{2}}$. Thus $Q(x)$ defined by (2.20) satisfies

$$
r|Q(x)|=|a|+\sqrt{{\overline{b_{1}}}^{2}+{b_{2}}^{2}} .
$$

Let $b_{2}=0, b_{1}=1 / 2$ and $a>0$. Then $r|Q(x)|=a+1 / 2$ and (2.29) does not hold for $k=-1$ so that $\dot{H}$ is not essentially selfadjoint by virtue of Theorem 2.7 , which with the next theorem yields the result.

Now, let us consider (P. 3).

Theorem 2.8. $\dot{H}$ with $Q$ given by (2.20) has a selfadjoint extension.

Proof. Let $\mathscr{D}$ be the totality of finite linear combinations of $U_{k, m}(\phi)$ (see (2.22)) with $\phi \in\left[C_{0}^{\infty}\left(\boldsymbol{R}_{+}\right)\right]^{2}$ and $\stackrel{H}{H}$ be the restriction of $H$ to $\mathscr{D}$. It is obvious that $\dot{H} \subset \dot{H} \subset \dot{H}$, so that $\dot{H}=\dot{H}$, which implies that $\dot{H}$ has a selfadjoint extension if and only if $\stackrel{H}{H}$ does. We define the operator $J$ by $J: U_{k, m}(\phi) \mapsto U_{k, m}(\bar{\phi})$. Then $J$ can be extended uniquely to a conjugation on $\mathscr{K}$. This conjugation commutes with $\dot{H}$ since the coefficients of $L(k)$ are real valued functions so that $\stackrel{H}{H}$ has a selfadjoint extension.

By the way, let us give a theorem which guarantees the existence of a selfadjoint extension of a certain type of $\dot{H}$ with $Q$ not necessarily given by (2.20). For a $4 \times 4$ matrix $A$ we denote by $\bar{A}$ the matrix whose $(j, k)$-element is the complex conjugate of the $(j, k)$-element of $A$ in the remaining part of this section only. Mimicking Veselić [16]; Lemma 1, we have

Theorem 2.9. Represent the matrices in terms of the basis in $C^{4}$ introduced 
in Lemma 2.1. Assume that $Q(x)$ satisfies

$$
A\left(\varepsilon_{1}, \varepsilon_{2}, \varepsilon_{3}\right) \overline{Q\left(\varepsilon_{1} x_{1}, \varepsilon_{2} x_{2}, \varepsilon_{3} x_{3}\right)}=Q\left(x_{1}, x_{2}, x_{3}\right) A\left(\varepsilon_{1}, \varepsilon_{2}, \varepsilon_{3}\right)
$$

for some $\varepsilon_{1}, \varepsilon_{2}, \varepsilon_{3}= \pm,\left(\varepsilon_{1}, \varepsilon_{2}, \varepsilon_{3}\right) \neq(+,+,+)$, where $A\left(\varepsilon_{1}, \varepsilon_{2}, \varepsilon_{3}\right)$ are constant matrices defined by

$$
\left\{\begin{array}{l}
A(+,+,-)=\alpha_{2} \alpha_{3} \beta \\
A(+,-,+)=\beta \\
A(+,-,-)=c_{1} \alpha_{3}+c_{2} \alpha_{1} \alpha_{2} \\
A(-,+,+)=\alpha_{1} \alpha_{2} \beta \\
A(-,+,-)=c_{1} I+c_{2} \alpha_{1} \alpha_{2} \alpha_{3} \\
A(-,-,+)=c_{1} \alpha_{1}+c_{2} \alpha_{2} \alpha_{3} \\
A(-,-,-)=\alpha_{2} \beta
\end{array}\right.
$$

where $c_{1}$ and $c_{2}$ are such constants that guarantee the unitarity of $A$ 's. Then $\dot{H}$ has a selfadjoint extension.

Proof. (2.3) with (2.4) implies

$$
\begin{aligned}
& \bar{\alpha}_{1}={ }^{t} \alpha_{1}=\alpha_{1}, \quad \bar{\alpha}_{2}={ }^{t} \alpha_{2}=-\alpha_{2}, \quad \bar{\alpha}_{2}={ }^{t} \alpha_{3}=\alpha_{3}, \\
& \bar{\beta}={ }^{t} \beta=\beta,
\end{aligned}
$$

which with (1.2) shows that each $A$ satisfies

$$
A={ }^{t} A \text {. }
$$

We define the operator $J$ by $J: \phi \mapsto A\left(\varepsilon_{1}, \varepsilon_{2}, \varepsilon_{3}\right) \overline{\psi\left(\varepsilon_{1} x_{1}, \varepsilon_{2} x_{2}, \varepsilon_{3} x_{3}\right)}$, which is a conjugation by virtue of the unitarity of $A$ and (2.33). $\dot{H}-\beta$ commutes with $J$ if

$$
A\left(\varepsilon_{1}, \varepsilon_{2}, \varepsilon_{3}\right) \bar{\alpha}_{j}=-\varepsilon_{j} \alpha_{j} A\left(\varepsilon_{1}, \varepsilon_{2}, \varepsilon_{3}\right) \quad(j=1,2,3)
$$

and (2.30) hold. The latter is assumed now. We can ascertain one by one that each $A$ defined by (2.31) satisfies (2.34) using (2.32) and (1.2). This completes the proof.

Another proof of Theorem 2.8 using Theorem 2.9. This $Q$ satisfies (2.30) for any $A$ in (2.31) commuting with $\beta$, since (2.34) implies $A \overline{i \alpha_{r}\left(\varepsilon_{1} x_{1}, \varepsilon_{2} x_{2}, \varepsilon_{3} x_{3}\right)}=$ $i \alpha_{r}\left(x_{1}, x_{2}, x_{3}\right) A$.

\section{$\S 3 . \quad$ Essential Selfadjointness}

Let us consider more general potentials than those in Section 2. Let $b_{1}, b_{2}$, and $s$ be real numbers. We put

$$
\begin{aligned}
A\left(b_{1}, b_{2}, s\right) & =A\left(x ; b_{1}, b_{2}, s\right) \\
& =\frac{i}{r} \alpha_{r} \beta b_{1}+\frac{1}{r} \beta b_{2}+\frac{i}{r} \alpha_{r} s, \\
(r & \left.=|x|, \quad \alpha_{r}=\sum_{j=1}^{3} \alpha_{\jmath} x_{\jmath} / r\right)
\end{aligned}
$$




$$
V\left(b_{1}, b_{2}, s\right)=V\left(x ; b_{1}, b_{2}, s\right)=Q(x)-A\left(x ; b_{1}, b_{2}, s\right),
$$

and

$$
m\left(b_{1}, b_{2}, s\right)=\operatorname{Min}_{k \in Z \backslash\{0\}} \sqrt{\left(k+b_{1}\right)^{2}+b_{2}{ }^{2}}+s-\frac{1}{2},
$$

where $\mathbb{Z}$ is the totality of integers. The condition of Theorem 2.7 is equivalent to the inequality

$$
a^{2} \leqq \operatorname{Min}_{k \in Z \backslash\{0\}}\left(k+b_{1}\right)^{2}+b_{2}{ }^{2}-\frac{1}{4} .
$$

So, it might be conjectured that the estimate

$$
r^{2} V\left(b_{1}, b_{2}, 0\right)^{2} \leqq m_{0}{ }^{2}<\operatorname{Min}_{k \in Z \backslash\{0\}}\left(k+b_{1}\right)^{2}+b_{2}{ }^{2}-\frac{1}{4}
$$

with some real constants $b_{1}, b_{2}$ and $m_{0}$ would imply the essential selfadjointness of $H$ with the potential $Q$. It has not yet been proved. (C.f. Corollary 3.2.) Our main result is

Theorem 3.1. (1) Assume that there exist real numbers $b_{1}, b_{2}, s$ and $m_{0}$ satisfying the following conditions:

$$
\begin{gathered}
|s| \leqq 1 / 2, \\
m\left(b_{1}, b_{2}, s\right)>0, \\
r^{2} V\left(b_{1}, b_{2}, s\right) * V\left(b_{1}, b_{2}, s\right) \leqq m_{0}^{2}<m\left(b_{1}, b_{2}, s\right)^{2} .
\end{gathered}
$$

(Here and in the sequel an inequality including operators is in the sense of quadratic form on $\dot{D} \times \dot{\mathscr{D}}$.) Then the operator $\dot{H}$ is essentially selfadjoint and the domain preserving property (1.5) holds.

(2) Assume

$$
m\left(b_{1}, b_{2}, 0\right)>0
$$

and

$$
r^{2} V\left(x ; b_{1}, b_{2}, 0\right)^{2} \leqq m\left(b_{1}, b_{2}, 0\right)^{2} .
$$

Then $\dot{H}$ is essentially selfadjoint.

Note that the assumptions of this theorem imply that

$$
|Q(x)| \leqq C_{1} / r
$$

for some constant $C_{1}$, so that $Q(x)$ satisfies $(1.4)^{\prime}$. Before proving this theorem we will give some applications. First, put $b_{1}=b_{2}=0$ and $s=\frac{1}{2}$. Then (3.5) and (3.6) are satisfied and (3.7) is nothing but (1.14), so that we have the result of [2] mentioned in Section 1. Secondly, we can give a partial answer to the conjecture mentioned above.

Corollary 3.2. Assume that there exist real constants $b_{1}, b_{2}$ and $m_{0}$ satisfying 
the following conditions:

(i) $\left(b_{1}, b_{2}\right) \neq(k, 0)$ for any $k \in \boldsymbol{Z} \backslash\{0\}$,

(ii) $V\left(b_{1}, b_{2}, 0\right)$ commutes with $\alpha_{r}$,

(iii) (3.4) holds.

Then $\dot{H}$ is essentially selfadjoint and (1.5) holds.

Proof. Let $s=\frac{1}{2}$. Then (3.5) and (3.6) are satisfied by virtue of (i), and $V\left(b_{1}, b_{2}, \frac{1}{2}\right)=V\left(b_{1}, b_{2}, 0\right)-\frac{i}{2 r} \alpha_{r}$ implies

$$
r^{2} V\left(b_{1}, b_{2}, \frac{1}{2}\right)^{*} V\left(b_{1}, b_{2}, \frac{1}{2}\right)=r^{2} V\left(b_{1}, b_{2}, 0^{2}\right)+\frac{1}{4}
$$

by virtue of (ii). Thus (3.4) implies (3.7) with $s=\frac{1}{2}$, so that we can apply Theorem 3.1(1) to obtain the result.

Corollary 3.3. (1) Assume that $Q(x)=Q_{0}(x)+Q_{1}(x), Q_{1}$ satisfying the conditions of Theorem 3.1(1) and $Q_{0}$ being of class $L_{2, \text { loc }}$ such that for any $\varepsilon>0$ and $R>0$ there exists a constant $C(\varepsilon, R)$ such that

$$
\left\|\chi_{R} Q_{0} u\right\| \leqq \varepsilon\left\|H_{0} u\right\|+C(\varepsilon, R)\|u\| \quad(\forall u \in \dot{D}),
$$

where $\chi_{R}$ is the characteristic fnnction of the ball $\left\{x \in \mathbb{R}^{3} ;|x| \leqq R\right\}$. Then $\dot{H}$ is essentially selfadjoint. In particular, if $C(\varepsilon, R)$ does not depend on $R$, then (1.5) holds.

(2) Assume that $Q(x)=\sum_{j=0}^{N} Q_{\jmath}\left(x-a_{\jmath}\right), Q_{0}$ being as above, each $Q_{j}(x)(1 \leqq j \leqq N)$ satisfying the assumptions of Theorem $3.1(1)$ and $a_{j}$ being distinct points in $\mathbb{R}^{3}$. Then $\dot{H}$ is essentially selfadjoint. If $C(\varepsilon, R)$ in (3.9) does not depend on $R$, then (1.5) holds.

Remark. As to sufficient conditions which guarantee (3.9), see e.g. ([2]; § 2).

Proof of Corollary 3.3. At first, let us prove (1). Put $H_{1}=H_{0}+Q_{1}$. Then $\dot{H}_{1}$ is essentially selfadjoint and $\overline{\dot{H}}_{0}\left(\vec{H}_{1}+i\right)^{-1}$ is bounded by virtue of Theorem 3.1(1) and the closed graph theorem. Thus (3.9) implies

$$
\begin{aligned}
\left\|\chi_{R} Q_{0} u\right\| & \leqq \varepsilon\left\|\bar{H}_{0}\left(\overline{\dot{H}}_{1}+i\right)^{-1}\left(\dot{H}_{1}+i\right) u\right\|+C(\varepsilon, R)\|u\| \\
& \leqq \varepsilon\left\|\bar{H}_{0}\left(\bar{H}_{1}+i\right)^{-1}\right\|\left\|\dot{H}_{1} u\right\|+\left[\varepsilon\left\|\bar{H}_{0}\left(\bar{H}_{1}+i\right)^{-1}\right\|+C(\varepsilon, R)\right]\|u\|, \\
& \text { for any } u \in \dot{\mathscr{D}} .
\end{aligned}
$$

Let $\varepsilon$ be so small that one can apply Lemma 1.3 to obtain that $\dot{H}_{0}+Q_{1}+\chi_{R} Q_{0}$ is essentially selfadjoint. Since $Q_{1} \in L_{2, \text { loc }}$ as was noted after Theorem 3.1, we can apply Lemma 1.2 to obtain the first assertion. If $C(\varepsilon, R)$ does not depend on $R$, then (3.10), Lemma 1.3(2) and Theorem 3.1(1) yield (1.5).

Next, let us prove (2). (3.8) which holds for $Q_{j}(1 \leqq j \leqq N)$ implies that 
$Q_{j}\left(x-a_{j}\right)$ is bounded outside a neighborhood of $a_{j}$. Thus the first half is an immediate consequence of Lemma 1.2 and (1). We can apply Landgren and Rejto's method [8] to obtain the second assertion.

Now let us prepare some lemmas to prove Theorem 3.1. We define the operators $H_{0 \pm}$ and $S_{ \pm}\left(b_{1}, b_{2}, s\right)$ by

$$
\begin{gathered}
H_{0 \pm}=H_{0}-\beta \pm i, \\
S_{ \pm}\left(b_{1}, b_{2}, s\right)=H_{0 \pm}+A\left(b_{1}, b_{2}, s\right) .
\end{gathered}
$$

Lemma 3.4. Assume (3.5) and (3.6). Then $S_{ \pm}=S_{ \pm}\left(b_{1}, b_{2}, s\right)$ satisfy

$$
S_{ \pm} * S_{ \pm} \geqq r^{-2} m\left(b_{1}, b_{2}, s\right)^{2}+\left(1-4 s^{2}\right) .
$$

If $s= \pm 1 / 2$, we also have

$$
S_{ \pm}{ }^{*} S_{ \pm} \geqq r^{-2}\left[m^{2}-\varepsilon / 4+\varepsilon(r-1 / 2)^{2}\right] \quad\left(0 \leqq{ }^{\forall} \varepsilon \leqq 1\right) .
$$

Proof. Since

$$
S_{ \pm}=\alpha_{r} p_{r}+\frac{i}{r} \alpha_{r} \beta\left(K+b_{1}\right)+\frac{\beta}{r} b_{2}+\frac{i}{r} \alpha_{r} s \pm i
$$

by virtue of (2.19), (3.1) and (3.12), their formal adjoint is

$$
S_{ \pm}^{*}=\alpha_{r} p_{r}+\frac{i}{r} \alpha_{r} \beta\left(K+b_{1}\right)+\frac{\beta}{r} b_{2}-\frac{i}{r} \alpha_{r} s i,
$$

where we have used the fact that $K$ commutes with $\alpha_{r}$ and $\beta$, and $p_{r}$ with $\alpha_{r}$. Note that $p_{r} \frac{1}{r}-\frac{1}{r} p_{r}=i r^{-2}$. Direct calculation shows

$$
S_{ \pm} * S_{ \pm}=\left(p_{r}^{2}-\frac{1}{4 r^{2}}\right)+Z_{ \pm}
$$

where we put

$$
Z_{ \pm}=r^{-2}\left[\left\{\beta\left(K+b_{1}\right)+s-\frac{1}{2}\right\}^{2}+b_{2}{ }^{2}-2 i \alpha_{r} \beta\left(s-\frac{1}{2}\right) b_{2}\right] \pm 2 r^{-1} \alpha_{r} s+1 .
$$

Let $\phi(r)$ be a real valued smooth function of $r>0$. Then

$$
0 \leqq\left\|\left(p_{r}-i \phi\right) u\right\|^{2}=\left(p_{r}^{2} u, u\right)-\left(\phi^{\prime} u, u\right)+\left(\phi^{2} u, u\right) \quad(u \in \dot{D}),
$$

so that we have $p_{r}^{2} \geqq \phi^{\prime}-\phi^{2}$. Put $\phi(r)=-\frac{1}{2 r}+a$, where $a$ is a constant, to obtain

$$
p_{r}^{2}-\frac{1}{4 r^{2}} \geqq \frac{a}{r}-a^{2} .
$$

Lemma 2.2 shows that $Z_{ \pm}$are reduced by each subspace $\mathfrak{g}(k, m)$ and that they are represented there by the matrices 


$$
\left(\begin{array}{cc}
r^{-2}\left[\left(k+b_{1}+s-\frac{1}{2}\right)^{2}+b_{2}{ }^{2}\right]+1 & -(1-2 s) b_{2} r^{-2} \mp 2 i s r^{-1} \\
-(1-2 s) b_{2} r^{-2} \pm 2 i s r^{-1} & r^{-2}\left[\left(k+b_{1}-s+\frac{1}{2}\right)^{2}+b_{2}{ }^{2}\right]+1
\end{array}\right)
$$

in terms of the basis $\left\{\Phi^{( \pm)}(k, m)\right\}$. The smallest eigenvalue of them is

$$
1+r^{-2}\left[\left(k+b_{1}\right)^{2}+b_{2}{ }^{2}+\left(\frac{1}{2}-s\right)^{2}-2 \sqrt{\left[\left(k+b_{1}\right)^{2}+b_{2}{ }^{2}\right]\left(\frac{1}{2}-s\right)^{2}+s^{2} r^{2}}\right],
$$

which is equal to

$$
\begin{aligned}
r^{-2}[ & \left.\sqrt{\left(k+b_{1}\right)^{2}+b_{2}{ }^{2}}+s-\frac{1}{2}\right]^{2}+1-2|s| r^{-1} \\
& +2 r^{-2}\left[\sqrt{\left(k+b_{1}\right)^{2}+b_{2}{ }^{2}}\left(\frac{1}{2}-s\right)+|s| r-\sqrt{\left[\left(k+b_{1}\right)^{2}+b_{2}{ }^{2}\right]\left(\frac{1}{2}-s\right)^{2}+s^{2} r^{2}}\right] .
\end{aligned}
$$

The last term of this is non-negative by virtue of (3.5). The first term is estimated from below by $r^{-2} m\left(b_{1}, b_{2}, s\right)$ by virtue of (3.3) and (3.6). Thus, in view of (3.14) and (3.15), we have

$$
S_{ \pm} * S_{ \pm} \geqq r^{-2} m\left(b_{1}, b_{2}, s\right)+r^{-1}(a-2|s|)+\left(1-a^{2}\right) .
$$

Put $a=2|s|$ in (3.16) to obtain (3.13). If $s= \pm \frac{1}{2}$, adding (3.13) multiplied by $1-\varepsilon$ and (3.16) with $a=0$ multiplied by $\varepsilon$, we have (3.13)'.

The following lemma will be used repeatedly.

Lemma 3.5 (Kato [5]; p. 190 and p. 196). Let $T$ be a closable operator in $\mathscr{H}$ with its closure possessing a bounded inverse. Let $B$ be a closable operator in If such that $\mathscr{D}(T) \subset \mathscr{D}(B)$ and there exists a constant $\delta(0<\delta<1)$ such that

$$
\|B u\| \leqq \delta\|T u\| \quad \text { for any } u \in \mathscr{D}(T) .
$$

Then $T+B$ is also closable, $\overline{T+B}=\bar{T}+\bar{B}$ has a bounded inverse and $\mathscr{D}(\overline{T+B})=$ $\mathscr{D}(\bar{T})$.

Lemma 3.6. Let $b_{1}^{\prime}, b_{2}^{\prime}$ and $s^{\prime}$ be real numbers such that

(a) they satisfy (3.5) and (3.6) with $b_{1}, b_{2}$ and $s$ replaced by $b_{1}^{\prime}, b_{2}^{\prime}$ and $s^{\prime}$, respectively,

(b) $\dot{S}_{ \pm}\left(b_{1}^{\prime}, b_{2}^{\prime}, s^{\prime}\right)$ are closable operators with its closure possessing a bounded inverse,

(c) $m\left(b_{1}^{\prime}, b_{2}^{\prime}, s^{\prime}\right)>\sqrt{\left(b_{1}-b_{1}^{\prime}\right)^{2}+\left(b_{2}-b_{2}^{\prime}\right)^{2}}+\left|s-s^{\prime}\right|$. Then $S_{ \pm}\left(b_{1}, b_{2}, s\right)$ are also closable operators with its closure possessing a bounded inverse and it holds that

$$
\left.\mathscr{D}\left(\overline{\dot{S}_{ \pm}\left(b_{1}, b_{2}, s\right.}\right)\right)=\mathscr{D}\left(\overline{\dot{S}_{ \pm}\left(b_{1}^{\prime}, b_{2}^{\prime}, s^{\prime}\right)}\right) .
$$

Proof. By virtue of Lemma 3.5, it sufficies to show (3.18) with $T=$ $\dot{S}_{ \pm}\left(b_{1}^{\prime}, b_{2}^{\prime}, s^{\prime}\right)$ and $B=A\left(b_{1}-b_{1}^{\prime}, b_{2}-b_{2}^{\prime}, s-s^{\prime}\right)$, that is, to show 


$$
\begin{gathered}
A\left(b_{1}-b_{1}^{\prime}, b_{2}-b_{2}^{\prime}, s-s^{\prime}\right) * A\left(b_{1}-b_{1}^{\prime}, b_{2}-b_{2}^{\prime}, s-s^{\prime}\right) \\
\leqq \\
\delta^{2} S_{ \pm}\left(b_{1}^{\prime}, b_{2}^{\prime}, s^{\prime}\right) * S_{ \pm}\left(b_{1}^{\prime}, b_{2}^{\prime}, s^{\prime}\right)
\end{gathered}
$$

for some $\delta<1$. The identities

$$
A\left(\tilde{b}_{1}, \tilde{b}_{2}, \tilde{s}\right)^{*} A\left(\tilde{b}_{1}, \tilde{b}_{2}, \tilde{s}\right)=r^{-2}\left[\tilde{b}_{1}^{2}+\tilde{b}_{2}{ }^{2}+2 \beta\left(\tilde{b}_{1}+i \alpha_{r} \tilde{b}_{2}\right) \tilde{s}+\tilde{s}^{2}\right]
$$

and

show that

$$
\left(\tilde{b}_{1}+i \alpha_{r} \tilde{b}_{2}\right) *\left(\tilde{b}_{1}+i \alpha_{r} \tilde{b}_{2}\right)=\tilde{b}_{1}^{2}+\tilde{b}_{2}^{2}
$$

$$
A\left(\tilde{b}_{1}, \tilde{b}_{2}, \tilde{s}\right) * A\left(\tilde{b}_{1}, \tilde{b}_{2}, \tilde{s}\right) \leqq r^{-2}\left(\sqrt{\tilde{b}_{1}^{2}+\tilde{b}_{2}^{2}}+|\tilde{s}|\right)^{2},
$$

which with $\tilde{b}_{j}=b_{j}-b_{j}^{\prime}(j=1,2) \tilde{s}=s-s^{\prime}$, (3.13) of Lemma 3.4 and (c) imply (3.19) for some $\delta<1$. This proves the present lemma.

Lemma 3.7. Assume (3.5) and (3.6). Then $\dot{S}_{ \pm}\left(b_{1}, b_{2}, s\right)$ are closable operators with its closure possessing a bounded inverse and

$$
\mathscr{D}\left(\overline{\dot{S}_{ \pm}\left(b_{1}, b_{2}, s\right)}\right)=\mathscr{D}\left(\overline{\dot{H}}_{0}\right) \text {. }
$$

Proof. Put $X=\boldsymbol{R}^{2} \times[-1 / 2,+1 / 2]$ and induce a metric $d$ on $X$ by $d\left(P, P^{\prime}\right)$ $=\sqrt{\left(b_{1}-b_{1}^{\prime}\right)^{2}+\left(b_{2}-b_{2}^{\prime}\right)^{2}}+\left|s-s^{\prime}\right|$ for $P=\left(b_{1}, b_{2}, s\right)$ and $P^{\prime}=\left(b_{1}^{\prime}, b_{2}^{\prime}, s^{\prime}\right)$. Let $G$ be the totality of $\left(b_{1}, b_{2}, s\right) \in X$ satisfying (3.6). $G$ is connected and open in $X$ and contains the origin $(0,0,0)$. For any $\left(b_{1}, b_{2}, s\right) \in G$, draw a curve $C$ in $G$ from $(0,0,0)$ to $\left(b_{1}, b_{2}, s\right)$ and put

$$
d_{0}=d(C, \partial G)=\underset{\left(b_{1}^{\prime}, b_{2}^{\prime}, s^{\prime}\right) \in C}{\operatorname{Min}} m\left(b_{1}^{\prime}, b_{2}^{\prime}, s^{\prime}\right)>0,
$$

where $\partial G$ denotes the boundary of $G$ in $X$ (not in $\boldsymbol{R}^{3}$ ). The minimum in the above exists and is positive since $C$ is compact and $m$ is continuous. The compactness of $C$ implies that there exist finite points $P_{0}=(0,0,0), P_{1}, \cdots, P_{j}=$ $\left(b_{1}{ }^{(j)}, b_{2}{ }^{(j)}, s^{(j)}\right), \cdots, P_{N}=\left(b_{1}, b_{2}, s\right)$ on $C$ such that the open balls with centers $P_{j}$ and $P_{j+1}$ and radius $d_{0} / 2$ have non-void intersection, so that $d\left(P_{j}, P_{j+1}\right)<d_{0} \leqq$ $m\left(b_{1}{ }^{(j)}, b_{2}{ }^{(j)}, s^{(j)}\right)$ for $0 \leqq j \leqq N-1$. This means that (c) in Lemma 3.6 with $\left(b_{1}^{\prime}, b_{2}^{\prime}, s^{\prime}\right)=\left(b_{1}{ }^{(j)}, b_{2}{ }^{(j)}, s^{(j)}\right)$ and $\left(b_{1}, b_{2}, s\right)=\left(b_{1}{ }^{(j+1)}, b_{2}{ }^{(j+1)}, s^{(j+1)}\right)$ holds. (a) is obvious since $P_{j} \in C \subset G$. Thus the induction and Lemma 3.6 show that the present lemma is valid if (b) in Lemma 3.6 with $b_{1}^{\prime}=b_{2}^{\prime}=s^{\prime}=0$ holds, which is obvious since $\dot{S}_{ \pm}(0,0,0)=\dot{H}_{0}-\beta \pm i$ and $\dot{H}_{0}$ is essentially selfadjoint as is well known.

Proof of Theorem 3.1(1). Let $\chi_{R}$ be the characteristic function of the ball $\left\{x \in \mathbb{R}^{3} ;|x| \leqq R\right\}$. We split $H \pm i$ into three parts :

$$
\begin{aligned}
H \pm i= & {\left[H_{0}-\beta+A\left(b_{1}, b_{2}, s\right) \pm i\right] } \\
& +\left[\chi_{R}\left\{Q-A\left(b_{1}, b_{2}, 0\right)\right\}-A(0,0, s)\right] \\
& +\left[\left\{1-\chi_{R}\right\}\left\{Q-A\left(b_{1}, b_{2}, 0\right)\right\}+\beta\right] \\
\equiv & S_{ \pm}\left(b_{1}, b_{2}, s\right)+B_{1}\left(b_{1}, b_{2}, s\right)+B_{2}\left(b_{1}, b_{2}\right) .
\end{aligned}
$$

$B_{2}=B_{2}\left(b_{1}, b_{2}\right)$ is symmetric and bounded by (3.8). Thus $\dot{H}$ is essentially self- 
adjoint if $\dot{H}-B_{2}$ is, and thus if the closures of $\dot{S}_{ \pm}\left(b_{1}, b_{2}, s\right)+B_{1}\left(b_{1}, b_{2}, s\right)$ have a bounded inverse. This follows Lemmas 3.5 and 3.7 , if it holds that

$$
B_{1}\left(b_{1}, b_{2}, s\right) * B_{1}\left(b_{1}, b_{2}, s\right) \leqq \delta^{2} S_{ \pm}\left(b_{1}, b_{2}, s\right) * S_{ \pm}\left(b_{1}, b_{2}, s\right)
$$

for some $\delta<1$. In view of (3.1) and (3.2),

$$
B_{1}=\chi_{R} V\left(b_{1}, b_{2}, s\right)-\left(1-\chi_{R}\right) A(0,0, s),
$$

so that we have

$$
B_{1}^{*} B_{1} \leqq r^{-2}\left[\chi_{R} m_{0}^{2}+\left(1-\chi_{R}\right) s^{2}\right],
$$

where we used (3.7). If $|s|<1 / 2$, in view of (3.13) of Lemma 3.4 and (3.23), in order to prove (3.22) it is sufficient to show

$$
\chi_{R} m_{0}^{2}+\left(1-\chi_{R}\right) s^{2} \leqq \delta^{2}\left[m\left(b_{1}, b_{2}, s\right)^{2}+\left(1-4 s^{2}\right) r^{2}\right] \quad \text { in } \quad x \in \mathbb{R}^{3}
$$

for some $\delta<1$, which is obvious for large $R$ by virtue of (3.7) and $1-4 s^{2}>0$. If $|s|=1 / 2$, in view of $(3.13)^{\prime}$ of Lemma 3.4 and (3.23), to prove (3.22) it sufficies to show

$$
\chi_{R} m_{0}^{2}+\left(1-\chi_{R}\right) \frac{1}{4} \leqq \delta^{2}\left[m^{\prime}\left(b_{1}, b_{2}, s\right)^{2}-\varepsilon / 4+\varepsilon\left(r-\frac{1}{2}\right)^{2}\right] \quad \text { in } \quad x \in \mathbb{R}^{3}
$$

for some $\varepsilon(0<\varepsilon<1)$ and $\delta(0<\delta<1)$. Let $\varepsilon$ be so small that $m_{0}{ }^{2}<m\left(b_{1}, b_{2}, s\right)^{2}$ $-\varepsilon / 4$, which is possible by virtue of (3.7), and let $\delta$ be so near 1 that $m_{0}{ }^{2}<$ $\delta^{2}\left[m\left(b_{1}, b_{2}, s\right)^{2}-\varepsilon / 4\right]$. Then (3.24) holds in $|x| \leqq R$ for any $R$ and in $|x| \geqq R$ for large $R$. Thus we have proved (3.22) so that $\dot{H}$ is essentially selfadjoint. (1.5) follows from (3.20) of Lemma 3.7, (3.22) and Lemma 3.5.

Proof of Theorem 3.1(2). Note that $A \equiv A\left(b_{1}, b_{2}, 0\right)$ and $V \equiv V\left(b_{1}, b_{2}, 0\right)$ are symmetric. Lemma 3.7 implies that $\dot{H}_{0}+A-\beta=\dot{S}_{ \pm}\left(b_{1}, b_{2}, 0\right) \mp i$ is essentially selfadjoint. (3.7)' and (3.13) of Lemma 3.4 show that

$$
\begin{aligned}
V^{2} & \leqq r^{-2} m\left(b_{1}, b_{2}, 0\right)^{2} \leqq \dot{S}_{ \pm}\left(b_{1}, b_{2}, 0\right) * S_{ \pm}\left(b_{1}, b_{2}, 0\right)-1 \\
& =\left(\dot{S}_{ \pm}\left(b_{1}, b_{2}, 0\right) \mp i\right) *\left(\dot{S}_{ \pm}\left(b_{1}, b_{2}, 0\right) \mp i\right),
\end{aligned}
$$

so that we can apply Lemma $1.3(1)$ to obtain that $\dot{S}_{ \pm}\left(b_{1}, b_{2}, 0\right) \mp i+V=\dot{H}_{0}+Q-\beta$ is essentially selfadjoint and so is $\dot{H}=\dot{H}_{0}+Q$.

\section{§4. Distinguished Selfadjoint Extensions and Invariance of the Essential Spectrum}

In this section we shall consider (P.4) and (P. 5). Schmincke [14] and Wüst [18] [19] have constructed a selfadjoint extension $\tilde{H}$ of $\dot{H}$ called distinguished, which has the property that all states in $\mathscr{D}(\widetilde{H})$ have finite potential energy:

$$
\mathscr{D}(\tilde{H}) \subset \mathscr{D}\left(r^{-1 / 2}\right) \text {; }
$$

on the other hand, Nenciu [9] has called $\tilde{H}$ distinguished when all states in $\mathscr{D}(\tilde{H})$ have finite kinetic energy: 


$$
\mathscr{D}(\widetilde{H}) \subset \mathscr{D}\left(\left|\bar{H}_{0}\right|^{1 / 2}\right)
$$

and has shown the unique existence of such an extension. Klaus and Wüst [6] have shown under an appropriate condition that these definitions coincide. Klaus and Wüst [7] have also obtained the invariance of essential spectrum. Their assumptions are respectively different and it is difficult to state them shortly. We note only that in the simplest case (1.8) all of them are reduced to the same condition $|e|<1$. (Cf. Example after Theorem 4.1.) The all authors mentioned above except Nenciu [9] treated the case of scalar potentials.

Our aim is to consider these problems in the case of the matrix potentials and to prove the following theorem.

Theorem 4.1. Assume that there exist constants $b_{1}, b_{2}, s, \sigma$ and $m_{0}$ such that they satisfy (3.5), (3.6),

$$
0 \leqq \sigma<\frac{1}{2}
$$

and

$$
r^{2} V\left(b_{1}, b_{2}, s-\sigma\right)^{*} V\left(b_{1}, b_{2}, s-\sigma\right) \leqq m_{0}^{2}<m\left(b_{1}, b_{2}, s\right)^{2} .
$$

(As to the notations, cf. (3.1)-(3.3).) Then (i) we have

$$
\mathscr{D}\left(\dot{H}^{*}\right) \cap \mathscr{D}\left(r^{-1 / 2}\right)=\mathscr{D}\left(\dot{H}^{*}\right) \cap \mathscr{D}\left(\left|\bar{H}_{0}\right|^{1 / 2}\right) .
$$

(ii) The restriction of $\dot{H}^{*}$ to the above domain, which will be denoted by $\tilde{H}$, is a selfadjoint extension of $\dot{H}$.

(iii) Let $\tilde{H}^{\prime}$ be a selfadjoint extension of $\dot{H}$ whose domain is contained in $\mathscr{D}\left(r^{-1 / 2}\right)$ or in $\mathscr{D}\left(\left|\bar{H}_{0}\right|^{1 / 2}\right)$. Then $\tilde{H}^{\prime}=\tilde{H}$.

(iv) $\sigma_{\text {ess }}(\widetilde{H})=\sigma_{\text {ess }}\left(\dot{H}_{0}\right)=R \backslash(-1,+1)$.

Remark. Assume $\sigma=0$. Then the assumptions of Theorem 4.1 is the same as those of Theorem 3.1(1), so that we have $\widetilde{H}=\bar{H}$ in this case.

Example. Assume that

$$
r|Q(x)| \leqq m_{0}<1
$$

Put $b_{1}=b_{2}=0$ and $s=\sigma$. Then $V(0,0,0)=Q$ and $m=1 / 2+\sigma$, so that (4.2) is satisfied for $\sigma$ sufficiently near $1 / 2$. The other assumptions of Theorem 4.1 are obviously satisfied in this case. (Cf. [9].)

We denote by $G$ the multiplication operator $r^{\sigma} \times$ in $\mathscr{H}$. Then $G=\dot{G}$ and $\dot{G}$ maps $\dot{D}$ onto itself bijectively. We put $\hat{T}=G T \dot{G}$ for any operator $T$ in $\mathscr{H}$. It is easy to see that $\hat{H}_{0}=\left(H_{0}+\frac{i}{r} \alpha_{r} \sigma\right) \dot{G}^{2}$ and

$$
\hat{A}\left(b_{1}, b_{2}, s\right)=A\left(b_{1}, b_{2}, s\right) \dot{G}^{2}
$$

so that we have 


$$
\hat{S}_{ \pm}\left(b_{1}, b_{2}, s-\sigma\right)=\dot{S}_{ \pm}\left(b_{1}, b_{2}, s\right) \dot{G}^{2}
$$

Lemma 4.2. Assume (3.5), (3.6) and (4.1). Then there exists a positive constant $C$ depending on $b_{1}, b_{2}, s$ and $\sigma$ such that

$$
\begin{aligned}
& \hat{S}_{ \pm}\left(b_{1}, b_{2}, s-\sigma\right) * \hat{S}_{ \pm}\left(b_{1}, b_{2}, s-\sigma\right) \geqq C\left(r^{4 \sigma-2}+r^{4 \sigma}\right), \\
& \begin{cases}\text { (a) }\left\|\hat{S}_{ \pm}\left(b_{1}, b_{2}, s-\sigma\right) u\right\| \geqq C\|u\|, \\
\text { (b) }\left\|\hat{S}_{ \pm}\left(b_{1}, b_{2}, s-\sigma\right) u\right\| \geqq C\left\|\dot{G}^{2} u\right\| \quad(u \in \dot{D}) .\end{cases}
\end{aligned}
$$

Proof. (3.5), (3.6) and Lemma 3.4 imply

$$
\dot{S}_{ \pm}\left(b_{1}, b_{2}, s\right) * \dot{S}_{ \pm}\left(b_{1}, b_{2}, s\right) \geqq C\left(r^{-2}+1\right),
$$

for some positive constant $C$, which with (4.6) shows (4.7). (4.7) and (4.1) show (4.8).

Lemma 4.3. Assume (3.5), (3.6) and (4.1). Then $\hat{S}_{ \pm}\left(b_{1}, b_{2}, s-\sigma\right)$ are closable and its closures have a bounded inverse and it holds that

$$
\left.\mathscr{D}\left(\overline{\hat{S}_{ \pm}\left(b_{1}, b_{2}, s-\sigma\right.}\right)\right)=\mathscr{D}\left(\overline{\hat{S}_{ \pm}(0,0,-\sigma)}\right) .
$$

Proof. Using (4.5) and (4.6), an argument similar to the proof of Lemma 3.6 shows that it also holds with $\dot{S}_{ \pm}\left(b_{1}^{\prime}, b_{2}^{\prime}, s^{\prime}\right)$ and $\dot{S}_{ \pm}\left(b_{1}, b_{2}, s\right)$ replaced by $\hat{S}_{ \pm}\left(b_{1}^{\prime}, b_{2}^{\prime}, s^{\prime}-\sigma\right)$ and $\hat{S}_{ \pm}\left(b_{1}, b_{2}, s-\sigma\right)$, respectively. The argument in the proof of Lemma 3.7 reduces the proof of the present lemma to the case of $b_{1}=b_{2}=s=0$. Since $\dot{G}^{2}$ maps $\dot{D}$ onto itself and the range of $\dot{S}_{ \pm}(0,0,0)=\dot{H}_{0}-\beta \pm i$ is dense, so is the range of $\hat{S}_{ \pm}(0,0,-\sigma)=\dot{S}_{ \pm}(0,0,0) \dot{G}^{2}$ (see (4.6)). This fact and (4.8-a) of Lemma 4.2 show that $\hat{S}_{ \pm}(0,0,-\sigma)$ have the desired properties, which yields the present lemma.

Decompose $H \pm i$ as in (3.21) with $s$ replaced by $s-\sigma$ and put

$$
\begin{aligned}
H_{1 \pm}\left(b_{1}, b_{2}\right) & \equiv H \pm i-B_{2}\left(b_{1}, b_{2}\right) \\
& =S_{ \pm}\left(b_{1}, b_{2}, s-\sigma\right)+B_{1}\left(b_{1}, b_{2}, s-\sigma\right) .
\end{aligned}
$$

Lemma 4.4. Under the assumptions of Theorem 4.1, $\hat{H}_{1 \pm}\left(b_{1}, b_{2}\right)$ is closable with its closure possessing a bounded inverse and

$$
\left.\mathscr{D}\left(\overline{\hat{H}_{1 \pm}\left(b_{1}, b_{2}\right.}\right)\right)=\mathscr{D}\left(\overline{\hat{S}_{ \pm}(0,0,-\sigma)}\right) .
$$

Proof. An argument similar to the proof of (3.22) shows, by using (4.2) instead of (3.7), that

$$
\left\|B_{1}\left(b_{1}, b_{2}, s-\sigma\right) u\right\| \leqq \delta\left\|S_{ \pm}\left(b_{1}, b_{2}, s\right) u\right\| \quad(u \in \dot{D})
$$

for some $\delta<1$. Put $u=\dot{G}^{2} u$ and note (4.5) and (4.6) to obtain

$$
\left\|\hat{B}_{1}\left(b_{1}, b_{2}, s-\sigma\right) u\right\| \leqq \delta\left\|\hat{S}_{ \pm}\left(b_{1}, b_{2}, s-\sigma\right) u\right\| \quad(u \in \dot{\mathscr{D}}),
$$


which with Lemma 3.5, (4.10) and Lemma 4.3 yields the present lemma.

In the sequel, we denote $\hat{H}_{1 \pm}\left(b_{1}, b_{2}\right)$ and $\hat{S}_{ \pm}\left(b_{1}, b_{2}, s-\sigma\right)$ by $\hat{H}_{1 \pm}$ and $\hat{S}_{ \pm}(s-\sigma)$, respectively, in short.

Lemma 4.5. Under the assumptions of Theorem 4.1, there exists a positive constant $C$ depending on $b_{1}, b_{2}, s$ and $\sigma$ such that

$$
\left\{\begin{array}{l}
\text { (a) }\left\|G^{2} u\right\| \leqq C\left\|\hat{\hat{S}}_{ \pm}(s-\sigma) u\right\|, \\
\text { (b) }\left\|\widehat{\hat{S}}_{ \pm}(s-\sigma) u\right\| \leqq C\left\|\overline{\hat{H}}_{1 \pm} u\right\|, \\
\text { (c) }\|G u\|^{2} \leqq C\left\|\overline{\hat{H}}_{1 \pm} u\right\|\|u\| \quad\left(u \in \mathscr{D}\left(\overline{\hat{H}}_{1 \pm}\right)\right) .
\end{array}\right.
$$

Proof. (a) is an immediate consequence of (4.8-b) of Lemma 4.2 and the closability of $\hat{S}_{ \pm}(s-\sigma)$ (Lemma 4.3). (b) follows from the boundedness of $\overline{\hat{S}_{ \pm}(s-\sigma)} \overline{\hat{H}}_{1 \pm}^{-1}$, which follows from Lemma 4.4 and the closed graph theorem. (c) follows from (a), (b) and $\|G u\|^{2}=\left(G^{2} u, u\right) \leqq\left\|G^{2} u\right\|\|u\|$.

Lemma 4.6. Assume the assumptions of Theorem 4.1. Put

$$
\check{H}_{1 \pm}=G^{-1} \overline{\hat{H}}_{1 \pm} G^{-1} \text { 。 }
$$

Then

$$
\check{H}_{1 \pm} \subset G^{-1} \overline{G \dot{H}}_{1 \pm}{ }^{(*)}
$$

and $\check{H}_{1 \pm} \mp i$ coincide with each other, which will be denoted by $H_{1 d}$, and are essentially selfadjoint. The operator $H_{d}$ defined by

$$
H_{d}=H_{1 d}+B_{2}, \quad B_{2}=B_{2}\left(b_{1}, b_{2}\right)
$$

is an essentially selfadjoint extension of $\dot{H}$.

Proof. At first let us prove $(4.13)^{\prime}$ and coincidence of $\check{H}_{1 \pm} \mp i$. Note that $G \dot{H}_{1 \pm}$ is closable since $G \dot{H}_{1 \pm} \subset\left(\dot{H}_{1 \mp} \dot{G}\right)^{*}$. Let $u \in \mathscr{D}\left(\bar{H}_{1 \pm} G^{-1}\right)$. Then there exists a sequence $\left\{v_{n}\right\} \subset \mathscr{D}\left(\hat{H}_{1 \pm}\right)=\dot{D}$ such that

$$
\left\{\begin{array}{l}
\text { (a) } v_{n} \rightarrow G^{-1} u \\
\text { (b) } \hat{H}_{1 \pm} v_{n}=G \dot{H}_{1 \pm} \dot{G} v_{n} \rightarrow \bar{H}_{1 \pm} G^{-1} u \text {. }
\end{array}\right.
$$

(4.15) and (4.12-c) of Lemma 4.5 imply that $\left\{\dot{G} v_{n}\right\}$ converges to $G G^{-1} u=u$, which with (4.15-b) shows that $u \in \mathscr{D}\left(\overline{G \dot{H}}_{1 \pm}\right)$ and $\overline{G \dot{H}}_{1 \pm} u=\bar{H}_{1 \pm} G^{-1} u$ so that we have $(4.13)^{\prime}$. (4.15-b) and (4.12-a, b) of Lemma 4.5 imply that

$$
G^{2} v_{n} \rightarrow G^{2} G^{-1} u=G u \text {. }
$$

(4.15-b) and (4.16) imply that $\overline{\hat{H}}_{1 \mp} v_{n}=\hat{H}_{1 \pm} v_{n} \mp 2 i G^{2} v_{n} \rightarrow \overline{\hat{H}}_{1 \pm} G u \mp 2 i G u$, which with (4.15-a) implies that $G^{-1} u \in \mathscr{D}\left(\bar{H}_{1 \mp}\right)$ and

(*) We can prove that $\check{H}_{1 \pm}=G^{-1} \overline{G \dot{H}}_{1 \pm}$. But $(4.13)^{\prime}$ is sufficient for our purpose. 


$$
\overline{\hat{H}}_{1 \mp} G^{-1} u=\overline{\hat{H}}_{1 \pm} G u \mp 2 i G u .
$$

Assume moreover that $u \in \mathscr{D}\left(\check{H}_{1 \pm}\right)\left(\subset \mathscr{D}\left(\overline{\hat{H}}_{1 \pm} G^{-1}\right)\right)$. Then the right hand side of (4.17) belongs to $\mathscr{D}\left(G^{-1}\right)$ and we have $\check{H}_{1 \pm} \mp i \subset \breve{H}_{1 \mp} \pm i$, and hence the coincidence of them.

$H_{1 d}$ is symmetric since $H_{1 d}=\check{H}_{1+}-i \subset\left(\check{H}_{1-}+i\right)^{*}=H_{1 d} *$. It is obvious that $\check{H}_{1 \pm} \supset \dot{H}_{1 \pm}$ so that $H_{d}$ is a symmetric extension of $\dot{H}$. Since $B_{2}$ is bounded and symmetric, $H_{d}$ is essentially selfadjoint if and only if so is $H_{1 d}$, or equivalently, if and only if the ranges of $H_{1 d} \pm i(\kappa+1)=\check{H}_{1 \pm} \pm i \kappa$ are dense in $\mathscr{A}$ for some constant $\kappa>0$. Let $\kappa$ be sufficiently small. Then in view of $(4.12-\mathrm{a}, \mathrm{b})$ of Lemmas 4.5 and 4.4 , we can apply Lemma 3.5 to obtain that the operators

$$
D_{ \pm} \equiv \overline{\hat{H}}_{1 \pm} \pm i \kappa G^{2}
$$

are closed and have the property

$$
\left\{\begin{array}{l}
\text { (a) } \mathscr{R}\left(D_{ \pm}\right)=\mathscr{H} \\
\text { (b) } \mathscr{D}\left(D_{ \pm}\right)=\mathscr{D}\left(\overline{\hat{H}}_{ \pm}\right) \supset \dot{D} .
\end{array}\right.
$$

(4.12-c) of Lemma 4.5 and (4.18-b) show that

$$
\mathscr{R}\left(G^{-1}\right)=\mathscr{D}(G) \supset \mathscr{D}\left(\overline{\hat{H}}_{1 \pm}\right)=\mathscr{D}\left(D_{ \pm}\right),
$$

which with (4.18) implies

$$
\mathscr{R}\left(G^{-1} D_{ \pm} G^{-1}\right)=\mathscr{R}\left(G^{-1}\right) \supset \mathscr{D}\left(D_{ \pm}\right) \supset \dot{D} .
$$

On the other hand, $G^{-1} D_{ \pm} G^{-1}=G^{-1} \widehat{H}_{1 \pm} G^{-1} \pm i \kappa=\check{H}_{1 \pm} \pm i \kappa$, so that we have $\mathscr{R}\left(\check{H}_{1 \pm} \pm i \kappa\right) \supset \dot{\mathscr{D}}$, which is dense in $\mathscr{K}$. This proves the present lemma.

Proof of Theorem 4.1. We shall prove later

$$
\mathscr{D}\left(\bar{H}_{d}\right)=\mathscr{D}\left(\dot{H}^{*}\right) \cap \mathscr{D}\left(r^{-1 / 2}\right)
$$

and

$$
\mathscr{D}\left(\bar{H}_{d}\right) \subset \mathscr{D}\left(\dot{H}^{*}\right) \cap \mathscr{D}\left(\left|\bar{H}_{0}\right|^{1 / 2}\right) .
$$

On the other hand, it is known $([5]$; p. 307$)$ that

$$
\mathscr{D}\left(\left|\bar{H}_{0}\right|^{1 / 2}\right) \subset \mathscr{D}\left(r^{-1 / 2}\right) \text {. }
$$

The above three formulas imply (i), (ii) and $\widetilde{H}=\bar{H}_{d}$ whose selfadjointness is already proved in Lemma 4.6. The assumption in (iii) implies $\tilde{H}^{\prime} \subset \widetilde{H}$, since a selfadjoint extension of $\dot{H}$ is a restriction of $\dot{H}^{*}$. The fact that a selfadjoint operator is maximal symmetric reduces this inclusion to the equality. Thus we obtain (iii). (iv) will be proved after proving (4.19) and (4.20).

At first, let us prove

$$
\mathscr{D}\left(\bar{H}_{d}\right) \subset \mathscr{D}\left(\dot{H}^{*}\right) \cap \mathscr{D}\left(r^{-1 / 2}\right) .
$$

There exists a positive constant $C$ such that 


$$
1 \leqq C\left(r^{2 \sigma-1}+r^{2 \sigma+1}\right) \quad \text { in } \quad r \geqq 0
$$

by (4.1) so that we have

$$
\left\|r^{-1 / 2} u\right\|^{2} \leqq C\left(\left\|r^{\sigma-1} u\right\|^{2}+\left\|r^{\sigma} u\right\|^{2}\right) \quad(u \in \dot{D}) .
$$

On the other hand (4.7) of Lemma 4.2 implies

$$
\left\|r^{\sigma-1} u\right\|^{2}+\left\|r^{\sigma} u\right\|^{2} \leqq C\left\|\hat{S}_{ \pm}(s-\sigma) \dot{G}^{-1} u\right\|^{2} \quad(u \in \dot{D})
$$

with some constant $C>0$. These two inequalities and (4.12-b) of Lemma 4.5 imply that

so that we have

$$
\left\|r^{-1 / 2} u\right\| \leqq C\left\|\hat{H}_{1 \pm} \dot{G}^{-1} u\right\|=C\left\|G \dot{H}_{1 \pm} u\right\| \quad(u \in \dot{\mathscr{D}}),
$$

$$
\left\|r^{-1 / 2} u\right\| \leqq C\left\|\overline{G \dot{H}}_{1 \pm} u\right\|, \quad\left(u \in \mathscr{D}\left(\overline{G \dot{H}}_{1 \pm}\right)\right) .
$$

Let $g(r)$ be $C^{\infty}$ function of $r>0$ such that $0 \leqq g(r) \leqq \operatorname{Min}\left(1, r^{-\sigma}\right)$ in $r \geqq 0, g(r) \equiv 1$ in $0 \leqq r \leqq 1 / 2$ and $g(r)=r^{-\sigma}$ in $r \geqq 2$. For any $u \in \mathscr{D}\left(\overline{G \dot{H}}_{1 \pm}\right)$, we have $g u \in \mathscr{D}\left(\overline{G \dot{H}}_{1 \pm}\right)$ and

$$
\bar{G}_{\dot{H}_{1 \pm}}(g u)=g \overline{G \dot{H}}_{1 \pm} u-i \alpha_{r} r^{\sigma} g^{\prime} u
$$

by virtue of the boundedness of $g$ and $r^{\sigma} g^{\prime}$. Now, using (4.23), (4.24) and (4.13)' of Lemma 4.6, we have

$$
\begin{aligned}
\left\|r^{-1 / 2} u\right\| & \leqq\left\|r^{-1 / 2} g u\right\|+\left\|r^{-1 / 2}(1-g) u\right\| \\
& \leqq C\left\|\bar{G}_{1 \pm}(g u)\right\|+\left\|r^{-1 / 2}(1-g) u\right\| \\
& \leqq C\left[\left\|G^{-1} \overline{G \dot{H}_{1 \pm}} u\right\|+\left\|r^{\sigma} g^{\prime} u\right\|\right]+\left\|r^{-1 / 2}(1-g) u\right\| \\
& \leqq C\left\|\check{H}_{1 \pm} u\right\|+C^{\prime}\|u\| \\
& \leqq C\left\|H_{d} u\right\|+C^{\prime \prime}\|u\|, \quad\left(u \in \mathscr{D}\left(\check{H}_{1 \pm}\right)=\mathscr{D}\left(H_{d}\right)\right),
\end{aligned}
$$

which implies $\mathscr{D}\left(\bar{H}_{d}\right) \subset \mathscr{D}\left(r^{-1 / 2}\right)$, and hence (4.22) since $\dot{H} \subset \bar{H}_{d} \subset \dot{H}^{*}$.

Next, let us prove that the restriction of $\dot{H}^{*}$ to $\mathscr{D}\left(\dot{H}^{*}\right) \cap \mathscr{D}\left(r^{-1 / 2}\right)$ is symmetric, or equivalently, that

$$
\operatorname{Im}\left(\dot{H}^{*} u, u\right)=0 \quad \text { for any } \quad u \in \mathscr{D}\left(\dot{H}^{*}\right) \cap \mathscr{D}\left(r^{-1 / 2}\right) .
$$

Then, in view of (4.22) and selfadjointness of $\bar{H}_{d}$, we have (4.19). It is known (see [4]; Lemma 9) that

$$
\mathscr{D}\left(\dot{H}^{*}\right)=\left\{u \in \mathscr{H} ; u \in\left[H^{1}{ }_{\text {loc }}\left(\boldsymbol{R}^{3} \backslash O\right)\right]^{4}, H u \in \mathscr{M}\right\} .
$$

Integration by parts yields

$$
\begin{aligned}
\operatorname{Im}\left(\dot{H}^{*} u, u\right) & =\frac{1}{2 i}\left[\left(\dot{H}^{*} u, u\right)-\left(u, \dot{H}^{*} u\right)\right] \\
& =\lim _{\substack{\rho \rightarrow 0 \\
R \rightarrow \infty}} \frac{1}{2 i} \int_{\rho \leqq x_{1} \leqq R}[\langle H u, u\rangle-\langle u, H u\rangle] d x \\
& =-\frac{1}{2} \lim _{\substack{\rho \rightarrow 0 \\
R \rightarrow \infty}}\left[I_{R}-I_{\rho}\right],
\end{aligned}
$$


where we put

$$
I_{R}=\int_{|x|=R}\left\langle\alpha_{r} u, u\right\rangle d S, \quad I_{\rho}=\int_{|x|=\rho}\left\langle\alpha_{r} u, u\right\rangle d S .
$$

Since the first member of (4.27) exists, the limits $\lim _{\rho \rightarrow 0} I_{\rho}=I_{0}$ and $\lim _{R \rightarrow \infty} I_{R}=I_{\infty}$ exist separately. Assume $I_{0} \neq 0$. Then for a certain $\rho_{0}$ we have $\left|I_{\rho}\right| \geqq \frac{1}{2}\left|I_{0}\right|$ for $0 \leqq \rho \leqq \rho_{0}$ and

$$
\int_{|x|=\rho}|u(x)|^{2} d S \geqq \int_{|x|=\rho}\left\langle\alpha_{r} u, u\right\rangle d S \geqq \frac{1}{2}\left|I_{0}\right| \quad\left(0 \leqq \rho \leqq \rho_{0}\right) .
$$

This inequality with the assumption $u \in \mathscr{D}\left(r^{-1 / 2}\right)$ yields

$$
\infty>\left\|r^{-1 / 2} u\right\|^{2} \geqq \int_{|x| \leqq \rho_{0}}|x|^{-1}\left|u(x) i^{2} d x \geqq \frac{1}{2}\right| I_{0} \mid \int_{0}^{\rho_{0}} \rho^{-1} d \rho=\infty,
$$

which is a contradiction. Next, assume $I_{\infty} \neq 0$. Then for a certain $R_{0}>0$, we have

$$
0<\frac{1}{2}\left|I_{\infty}\right| \leqq\left|I_{R}\right| \leqq \int_{|x|=R}|u|^{2} d S \quad\left(R \geqq R_{0}\right) .
$$

Integration from $R_{0}$ to $\infty$ leads to a contradiction. Thus we have $I_{0}=I_{\infty}=0$, which with (2.27) yields (4.25), and hence (4.19).

Next, let us prove (4.20). By virtue of (4.26), (3.8), which is valid in view of (4.2), and (1.9), we have

$$
\mathscr{D}\left(\overline{\dot{H}}_{0}\right)=\left[H^{1}\right]^{4} \subset \mathscr{D}\left(\dot{H}^{*}\right) .
$$

On the other hand, (4.21) implies

$$
\mathscr{D}\left(\bar{H}_{0}\right) \subset \mathscr{D}\left(\left|\bar{H}_{0}\right|^{1 / 2}\right) \subset \mathscr{D}\left(r^{-1 / 2}\right),
$$

which with (4.19) and (4.28) implies

$$
\mathscr{D}\left(\bar{H}_{0}\right) \subset \mathscr{D}\left(\bar{H}_{d}\right) .
$$

Let $\kappa$ be a non zero real number. (4.19), (4.29), the closed graph theorem and (3.8) show the boundedness of the operators $r^{-1 / 2}\left(\bar{H}_{d} \pm i \kappa\right)^{-1}, r^{-1 / 2}\left(\bar{H}_{0} \pm i \kappa\right)^{-1}$, their adjoints

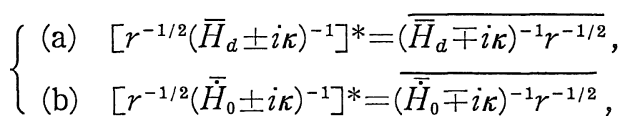

and $r Q$. The boundedness of these operators and (4.30) show the resolvent equation

$$
\begin{aligned}
& \left(\bar{H}_{d}-i \kappa\right)^{-1}-\left(\overline{\dot{H}}_{0}-i \kappa\right)^{-1} \\
& \quad=\left(\bar{H}_{d}-i \kappa\right)^{-1}\left(\bar{H}_{0}-i \kappa\right)\left(\overline{\dot{H}}_{0}-i \kappa\right)^{-1}-\left(\bar{H}_{d}-i \kappa\right)^{-1}\left(\bar{H}_{d}-i \kappa\right)\left(\overline{\dot{H}}_{0}-i \kappa\right)^{-1} \\
& \quad=-\left(\overline{\bar{H}}_{d}-i \kappa\right)^{-1} r^{-1 / 2} \cdot r Q \cdot\left\{r^{-1 / 2}\left(\bar{H}_{0}-i \kappa\right)^{-1}\right\} .
\end{aligned}
$$

Noting $\left(\overline{\left.\bar{H}_{d}-i \kappa\right)^{-1} r^{-1 / 2}} * r^{-1 / 2}\left(\bar{H}_{d}+i \kappa\right)^{-1}\right.$, which follows from (4.31-a), and (4.31-b), take the adjoint of the above expression to obtain 


$$
\left(\bar{H}_{d}+i \kappa\right)^{-1}=\left(\overline{\dot{H}}_{0}+i \kappa\right)^{-1}-\left(\overline{\left.\bar{H}_{0}+i \kappa\right)^{-1} r^{-1 / 2}} \cdot r Q \cdot\left\{r^{-1 / 2}\left(\bar{H}_{d}+i \kappa\right)^{-1}\right\} .\right.
$$

In view of the relations $\mathscr{R}\left(\left(\overline{\dot{H}}_{0}+i \kappa\right)^{-1}\right)=\mathscr{D}\left(\overline{\dot{H}}_{0}\right)=\mathscr{D}\left(\left|\vec{H}_{0}\right|\right) \subset \mathscr{D}\left(\left|\overline{\dot{H}}_{0}\right|^{1 / 2}\right)$ and $\mathscr{D}\left(\bar{H}_{d}\right) \subset$ $\mathscr{D}\left(\dot{H}^{*}\right)$, in order to show (4.20), it is sufficient to show

$$
\mathscr{R}\left(\left(\overline{\left.\overline{\dot{H}}_{0}+i \kappa\right)^{-1} r^{-1 / 2}}\right)\right) \subset \mathscr{D}\left(\left|\overline{\dot{H}}_{0}\right|^{1 / 2}\right) .
$$

The closure of $\left|\bar{H}_{0}\right|^{1 / 2}\left(\vec{H}_{0}+i \kappa\right)^{-1}\left|\bar{H}_{0}\right|^{1 / 2}$ is bounded, so that we have

$$
\overline{\left(\overline{\hat{H}}_{0}+i \kappa\right)^{-1} r^{-1 / 2}}=\left|\overline{\dot{H}}_{0}\right|^{-1 / 2} \cdot \mid \overline{\left.\overline{\dot{H}}_{0}\right|^{1 / 2}\left(\overline{\dot{H}}_{0}+i \kappa\right)^{-1}\left|\overline{\dot{H}}_{0}\right|^{1 / 2}} \cdot \overline{\left|\bar{H}_{0}\right|^{-1 / 2} r^{-1 / 2}},
$$

which implies (4.33), and hence (4.20).

By Weyl's theorem ([5]; Chap. IV Problem 5.38) and $\bar{H}_{d}=\widetilde{H}$, (iv) holds if the first factor of the second term of the right hand side of (4.32) or its adjoint $r^{-1 / 2}\left(\vec{H}_{0}-i \kappa\right)^{-1}$ is compact, since the other two factors there are bounded. Let $\chi_{R} \in C^{\infty}$ be such that $\chi_{R}(x)=1(|x| \leqq R), \chi_{R}(x)=0(|x| \geqq R+1)$ and $0 \leqq \chi_{R} \leqq 1$. It sufficies to show that $\chi_{R}(x) r^{-1 / 2}\left(\bar{H}_{0}-i \kappa\right)^{-1}$ is compact, since it tends to $r^{-1 / 2}\left(\bar{H}_{0}-i \kappa\right)^{-1}$ in operator norm as $R \rightarrow \infty$. The Hilbert space $\mathscr{H}=\left[L_{2}\right]^{4}$ is mapped boundedly by $\left(\dot{H}_{0}-i \kappa\right)^{-1}$ onto the Sobolev space $\left[H^{1}\right]^{4}$, which is mapped compactly by $\chi_{R}$ into $\left[L_{p}\right]^{4}(1<p<6)$ by virtue of the Theorem 2 of Sobolev [15]; $\S 11$. The last space is mapped boundedly by $\chi_{R+1} \gamma^{-1 / 2}$ into $\mathscr{H}$, if $\chi_{R+1} \gamma^{-1 / 2} \in L_{q} \quad(q \geqq 1$ and $\frac{1}{p}+\frac{1}{q}=2$ ), by virtue of Hölder's inequality. It is obvious that $\chi_{R+1} r^{-1 / 2} \in L_{q}$ for $q<6$ so that we can choose $p$ and $q$ satisfying the above conditions, which completes the proof.

In conclusion, the author wishes to express his sincere gratitude to Professor T. Ikebe for his enduring encouragements and valuable advices and to Professor O. Yamada for valuable discussions.

\section{References}

[1] Arai, M., On essential self-adjointness of Dirac operators, RIMS Kokyuroku, Kyoto Univ., 242 (1975), 10-21.

[2] Arai, M. and Yamada, O., Essential self-adjointness and invariance of the essential spectrum for Dirac operators, Publ. RIMS, Kyoto Univ. 18 (1982), 973-985.

[3] Chernoff, P.R., Schrödinger and Dirac operators with singular potentials and hyperbolic equations, Pacific J. Math., 72 (1977), 361-382.

[4] Evans, W.D., On the unique selfadjoint extension of the Dirac operator and the existence of the Green matrix, Proc. London Math. Soc., 20 (1970), 537-557.

[5] Kato, T., Perturbation Theory for Linear Operators, Springer, 1966.

[6] Klaus, M. and Wüst, R., Characterization and uniqueness of distinguished selfadjoint extensions of Dirac operators, Comm. math. Phys., 64 (1979), 171-176.

[7] - Spectral properties of Dirac operators with singular potetials, J. Math. Anal. Appl., 72 (1979), 206-214.

[8] Landgren, J. J. and Rejto, P.A., On a theorem of Jörgens and Chernoff concerning essential self-adjointness of Dirac operators, J. reine angew. Math., 332 (1981), $1-14$. 
[9] Nenciu, G., Self-adjointness and invariance of the essential spectrum for Dirac operators defined as quadratic forms, Comm. math. Phys., 48 (1976), 235-247.

[10] Rellich, F., Eigenwertheorie partieller Differentialgleichungen II, Vorlesungsmanuskript, Göttingen, 1953.

[11] — - Die zulässigen Randbedingungen bei den singulären Eigenwertproblemen der mathematischen Physik (Gewöhnliche Differentialgleichungen zweiter Ordnung), Math. Z., 49 (1943/44), 702-723.

[12] Roos, B.W. and Sangren, W.C., Spectral theory of Dirac's radial relativistic wave equations, J. Math. Phys., 3 (1962), 882-890.

[13] Schmincke, U.-W., Essential self-adjointness of Dirac operators with a strongly singular potential, Math. Z., 126 (1972), 71-81.

[14] - Distinguished selfadjoint extensions of Dirac operators, Math. Z., 129 (1972), 335-349.

[15] Sobolev, S.L., Applications of Functional Analysis in Mathematics, Leningrad, 1950, (English translation: A.M.S., 1963).

[16] Veselić, K., The nonrelativistic limit of the Dirac equation and the spectral concentration, Glasnik Math., 4(24) (1969), 231-240.

[17] Weidmann, J., Oszillationsmethoden für Systeme gewöhnlicher Differentialgleichungen, Math. Z., 119 (1971), 349-373.

[18] Wüst, R., Distinguished self-adjoint extensions of Dirac operators constructed by means of cut-off potentials, Math. Z., 141 (1975), 93-98.

[19] —, Dirac operators with strongly singular potentials-distinguished selfadjoint extensions constructed with a spectral gap theorem and cut-off potentials, Math. Z., 152 (1977), 259-271. 
